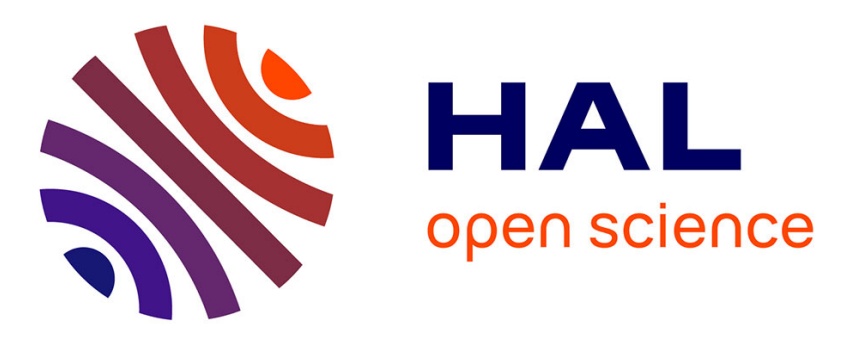

\title{
Elaboration d'une typologie des dépôts de blocs supratidaux de sommets de falaise de la péninsule de Reykjanes (Islande)
}

\author{
Ronan Autret, Serge S. Suanez, Bernard Fichaut, Samuel Etienne
}

\section{To cite this version:}

Ronan Autret, Serge S. Suanez, Bernard Fichaut, Samuel Etienne. Elaboration d'une typologie des dépôts de blocs supratidaux de sommets de falaise de la péninsule de Reykjanes (Islande). Géomorphologie: relief, processus, environnement, 2016, Analyses géomorphologiques d'événements basse fréquence haute énergie sur les versants, les dynamiques fluviales et les littoraux d'Islande et apports méthodologiques, 22 (1), pp.61-76. 10.4000/geomorphologie.11272 . hal-01258704

\author{
HAL Id: hal-01258704 \\ https://hal.science/hal-01258704
}

Submitted on 16 Jan 2018

HAL is a multi-disciplinary open access archive for the deposit and dissemination of scientific research documents, whether they are published or not. The documents may come from teaching and research institutions in France or abroad, or from public or private research centers.
L'archive ouverte pluridisciplinaire HAL, est destinée au dépôt et à la diffusion de documents scientifiques de niveau recherche, publiés ou non, émanant des établissements d'enseignement et de recherche français ou étrangers, des laboratoires publics ou privés. 


\title{
Elaboration d'une typologie des dépôts de blocs supratidaux de sommets de falaise de la péninsule de Reykjanes (Islande)
}

\section{Development of a typology of cliff-top storm deposits of the Rekjanes Peninsula (Iceland)}

\author{
Ronan Autret ${ }^{\star a}$, Serge Suanez $z^{\mathrm{a}}$, Bernard Fichaut ${ }^{\mathrm{a}}$, Samuel Etienne ${ }^{\mathrm{b}}$ \\ ${ }^{a}$ LETG Brest Géomer - UMR 6554 CNRS - Institut Universitaire Européen de la Mer - Plouzané - France. \\ bLETG Dinard - UMR 6554 CNRS - Laboratoire de Géomorphologie et Environnement Littoral, École Pratique des Hautes Études, 15, boulevard de la mer - Dinard - France.
}

\section{INFORMATION SUR L'ARTICLE}

Reçu le 25 juin 2015

Reçu sous sa forme révisée le 22 septembre 2015 Définitivement accepté le 14 décembre 2015

*Auteur correspondant. Tél : +33298498691; Fax : +33298498703

Courriels :

ronan.autret@univ-brest.fr (R. Autret) serge.suanez@univ-brest.fr (S. Suanez) bernard.fichaut@univ-brest.fr (B. Fichaut) samuel.etienne@ephe.sorbonne.fr (S. Etienne)

\begin{abstract}
RÉSUMÉ
Cette étude porte sur la répartition et la morphologie des dépôts grossiers de tempêtes $(25,6 \mathrm{~cm} \leq 1 \leq 820 \mathrm{~cm})$ qui se trouvent en retrait du sommet des falaises de la péninsule de Reykjanes, au sud-ouest de l'Islande. Les $35 \mathrm{~km}$ de linéaire côtier sur lesquels porte ce travail sont constitués de falaises, hautes au maximum de 15 mètres, taillées dans un substrat basaltique postglaciaire dâge compris entre 14500 et 800 ans BP. Lorigine de ces dépôts est marine et est attribuée à laction des vagues engendrées par les dépressions atlantiques, vagues dont les hauteurs significatives pour une période de retour de 100 ans atteignent 17,4 mètres dans ce secteur. Entre les mois d’avril et juin 2014, un travail de terrain a permis détudier 35 accumulations supratidales de blocs à partir d’observations qualitatives et de relevés topomorphologiques. L'analyse de ces données montre que l'altitude du sommet des falaises nést pas un facteur limitant à la formation de ces dépôts sur la péninsule de Reykjanes. Au contraire, le contexte lithostructural des coulées de lave et les spécificités hydrodynamiques locales qui influent sur la morphologie des falaises sont prédominantes dans la formation et la disposition des différents types d’accumulations.
\end{abstract}

Mots clés : blocs supratidaux, côte rocheuse, falaise, tempête, Islande.

\section{ABSTRACT}

The present study focuses on the distribution and morphology of cliff-top storm deposits (CTSD) in the Reykjanes Peninsula in southwest Iceland. The study area stretches over a 35 kilometre coastline and consists mainly of cliffs not exceeding $15 \mathrm{~m}$, cut into post-glacial lava beds aged 14500 to $800 \mathrm{yr}$ BP. The origin of these deposits cannot be related to tsunamis since none has been recorded in this area within this period. It is due to the action of North Atlantic waves whose significant height, measured between 1994 and 2004, can reach $17.4 \mathrm{~m}$ in this area. During a field survey from April to June, 2014, 35 CTSD sites were studied mainly through qualitative observations and topomorphological measurements. The analysis of the data shows that the organisation and distribution of CTSD in the Reykjanes peninsula do not depend on the cliff height. In contrast, the lithostructural characteristics of the lava beds and local variations in hydrodynamism, both of which determine the morphology of cliffs, appear to be decisive factors in explaining these aspects of CTSD in this area.

Key words: Cliff-top storm deposits (CTSD), rock coast, cliff, storm, Iceland.

\section{Introduction}

Les dépôts de blocs supratidaux, appelés le plus souvent boulders ou clasts dans la littérature anglophone (Hearty, 1997), ou blocs cyclopéens dans la littérature française (Fichaut et Suanez, 2008), se répartissent préférentiellement le long des côtes à falaises rocheuses faisant face à des eaux profondes et soumises à l'action de vagues géantes. Ils appartiennent à la famille des dépôts très grossiers (Terry et Goff, 2014) au même titre que les blocs erratiques ou les blocs glaciels. Si ces derniers relèvent respectivement de processus glaciaires en milieu continental (Charpentier, 1835) et glaciels en milieu marin (Dionne, 2001), les mégablocs supratidaux sont le résultat de dynamiques marines de très haute énergie (Noormets et al., 2004 ; Hansom et Hall, 2009). Leur mise en place est toujours sujette à un débat qui oppose une partie de la communauté scientifique privilégiant l'action des tsunamis, à celle qui rattache ces dynamiques aux évènements météo-océaniques extrêmes comme les tempêtes ou les différents types de cyclones tropicaux (ouragans, typhons, etc. ; Dawson et Shi, 2000 ; Nott, 1997, 2003).

Dans un premier temps, létude des mégablocs a eu pour objectif de caractériser des niveaux grossiers dans des stratigraphies anciennes du Pléistocène (Moore et Moore, 1984 ; Paskoff, 1991 ; Young et Bryant, 1992 ; Hearty, 1997 ; Hartley et al., 2001) et de l'Holocène (Dawson, 1999 ; Dawson et Shi, 2000 ; Nakata et Kawana, 1995). À partir des années 2000 principalement, ces travaux ont bien plus porté sur leur caractérisation morphosédimentaire et les processus hydrodynamiques à lorigine de leur mise en place.

Sur tout le pourtour de la façade atlantique nord-européenne, la majorité des travaux portant sur létude des mégablocs les ont classés dans la famille des cliff-top storm deposits (CTSD), terme qui pourrait se traduire par "dépôts de tempête de sommet de falaise ». Ces CTSD ont été identifiés sur les îles Orcades et Shetland au nord 
de l'Ecosse (Hall et al., 2006; Hansom et Hall, 2009, Scheffers et al., 2009), dans les îles d'Aran au large de la côte ouest de l'Irlande (Hall et al., 2006 ; Knight et al., 2009 ; Knight et Burningham, 2011 ; Scheffers et al., 2009 ; Williams et Hall, 2004 ; Cox et al., 2012), en mer d'Iroise, sur l'île de Banneg dans l'archipel de Molène
(Suanez et al., 2009 ; Fichaut et Suanez, 2011), en Islande sur la péninsule de Reykjanes (Etienne, 2007 ; Etienne et Paris, 2010).

Létude des mégablocs a fait l'objet d'analyses descriptives morphosédimentaires et dynamiques approfondies en fonction de la disposition, de la taille et de la morphométrie des blocs, ainsi que
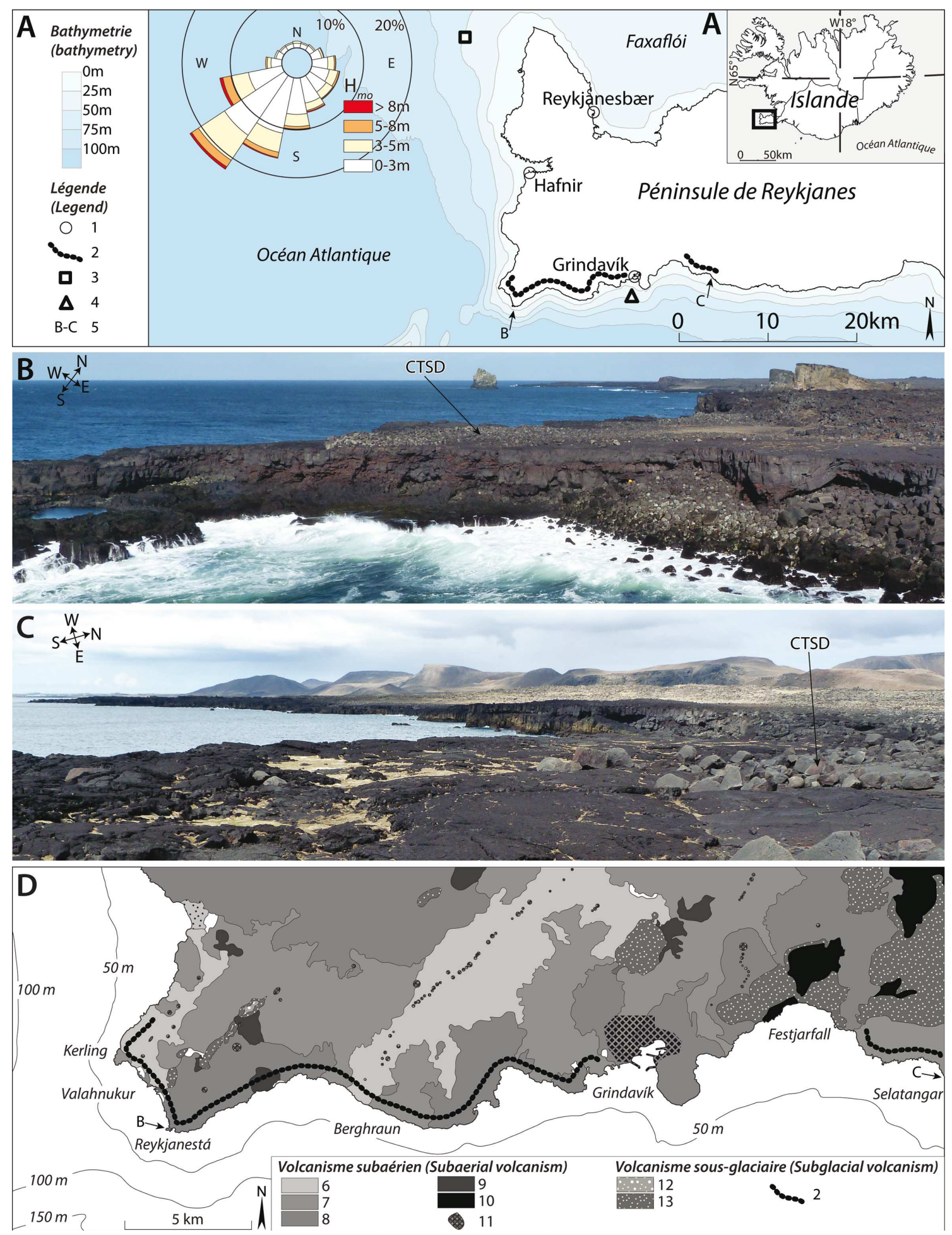

Fig. 1 - Localisation de la péninsule de Reykjanes et du site d'étude.

Échelle régionale (A). 1 : ville ; 2 : site d'étude ; 3 : bouée Gardaskaga ; 4 : Bouée Grindavik; 5 : localisation des photographies ci-dessous.

Photographies des sites de Reykjanestá (B) et de Selatangar (C), et carte géologique du site d'étude (D). 6 : XIIIème siècle ; $7: 2-3$ milliers d'années (ma) $; 8: 3-11,5$ ma $9: 12,5-14,5 \mathrm{ma} ; 10:$ coulées de laves interglaciaires antérieures ; $11:$ cône ; $12: 15$ $20 \mathrm{ma} ; 13: 20-45 \mathrm{ma}$.
Fig. 1 - Location of the Reykjanes Peninsula and the study area.

Regional scale (A). 1: town; 2: study area; 3: gardaskaga buoy; 4: grindavik buoy; 5: photograph locations.

Photographs of Reykjanestá Point (B) and Selatangar (C), and geological map of the southwest part of the Reykjanes Peninsula (D). 6: XIIIth century; 7: 2-3 ka: 8: 3-11.5 ka: 9: 12.5-14.5 ka; 10: oldest interglacial lavas; 11: cone; 12: 15-20 ka; 13: 20-45 ka. 
du contexte morphologique et hydrodynamique général de leur environnement côtier. La disposition stratigraphique des mégablocs dépend de la morphologie des fonds marins et de la côte, mais aussi des conditions lithostructurales et hydrodynamiques (Nott, 1997). La disposition la plus simple se présente sous la forme de blocs isolés, que les anglophones nomment « single, isolated ou individual boulder ou clast " (Jones et Hunter, 1992 ; Dawson et Shi, 2000 ; Whelan et Keating, 2004). Ils peuvent également se présenter sous la forme d'empilements ou d'amas anarchiques de blocs simplement imbriqués " imbricated boulders ou clasts ». La disposition la plus édifiante concerne les remparts ou barrages de blocs imbriqués que l'on trouve dans la littérature anglophone sous les termes de " imbricated boulder trains, continuous boulder ridges ou detrital boulder ridges » (Nott, 1997, 2003 ; Kelletat et Schellmann, 2002). Ces derniers sorganisent sous la forme d'alignements parallèles au trait de côte et sont généralement constitués de dalles (plutôt que de boules) adossées les unes aux autres en formant un tuilage. Dans certains cas, il existe des systèmes de barrages multiples ; ils prennent alors l'allure de rides successives, alignées parallèlement au trait de côte comme cela a été décrit dans l'archipel de Molène (Suanez et al., 2009). Les dispositions énumérées ci-dessus ont été décrites sur divers substrats, tels que des roches sédimentaires (par ex. calcaire carbonifère, grès, mudstone, marnes, etc. ; Williams et al., 2004 ; Hall et al., 2006 ; Scheffers et al., 2009 ; Kogure et Matsukura, 2010 ; Switzer et Burston, 2010 ; Shah-Hosseini et al., 2011, 2013 ; Cox et al., 2012 ; Salzmann et Green, 2012 ; Miller et al., 2013 ; Prizomwala et al., 2014) ou des roches magmatiques, volcaniques et plutoniques (par ex. basalte, ignimbrite, tuf, rhyolite, granite ; Hall et al., 2006 ; Hansom et al., 2008 ; Scheffers et al., 2009 ; Suanez et al., 2009 ; Knight et al., 2009 ; Etienne et Paris, 2010 ; Goto et al., 2011 ; Richmond et al., 2011).

Le travail présenté ici se situe dans la continuité de l'étude d'Etienne et Paris (2010) qui a porté sur la réalisation d'un inventaire descriptif des dépôts de tempêtes que l’on trouve sur la côte rocheuse volcanique de la péninsule de Reykjanes (fig. 1). Notre recherche, quant à elle, a consisté à établir une typologie de ces mégablocs (que nous appellerons CTSD), reposant sur un ensemble de critères lithostructuraux, morphologiques et sédimentaires propres à cet environnement côtier. Lanalyse descriptive et quantitative mise en œuvre repose sur des mesures topo-morphologiques et granulométriques réalisées sur plus d'une trentaine d'accumulations réparties sur $35 \mathrm{~km}$ de linéaire côtier. Elle nous a permis de distinguer et caractériser les différents secteurs propices à la mise en place de CTSD. Cette typologie repose enfin sur des critères dynamiques en matière d'arrachement, de déplacement et de dépôt de blocs, identifiés à partir d'une étude diachronique de photographies obliques de terrain.

\section{Aire étudiée}

\subsection{Contexte géomorphologique}

La péninsule de Reykjanes se situe au sud-ouest de l'Islande. Elle correspond à une zone de rifting, l'un des deux secteurs de divergence active des plaques tectoniques en Islande matérialisant la partie émergée de la dorsale médio-atlantique (fig. 1). Le volcanisme est quasi-continu à cet endroit depuis le Pléistocène moyen ; douze évènements éruptifs historiques (post-874) et au moins neuf éruptions préhistoriques ont été recensés depuis cette période (Thordarson et Larsen, 2007). La zone étudiée en détail se situe à l’extrémité sudouest de la péninsule de Reykjanes (fig. 1). Il s'agit d'un linéaire côtier de $35 \mathrm{~km}$, essentiellement constitué de falaises dont l'altitude sommitale est comprise entre 4 et 18 mètres. Hormis le promontoire en hyaloclastite de Valahnukur et les falaises composites de Karl et de Festjarfall, les falaises de la péninsule de Reykjanes sont taillées dans un substrat basaltique issu d'un volcanisme subaérien (fig. 1).
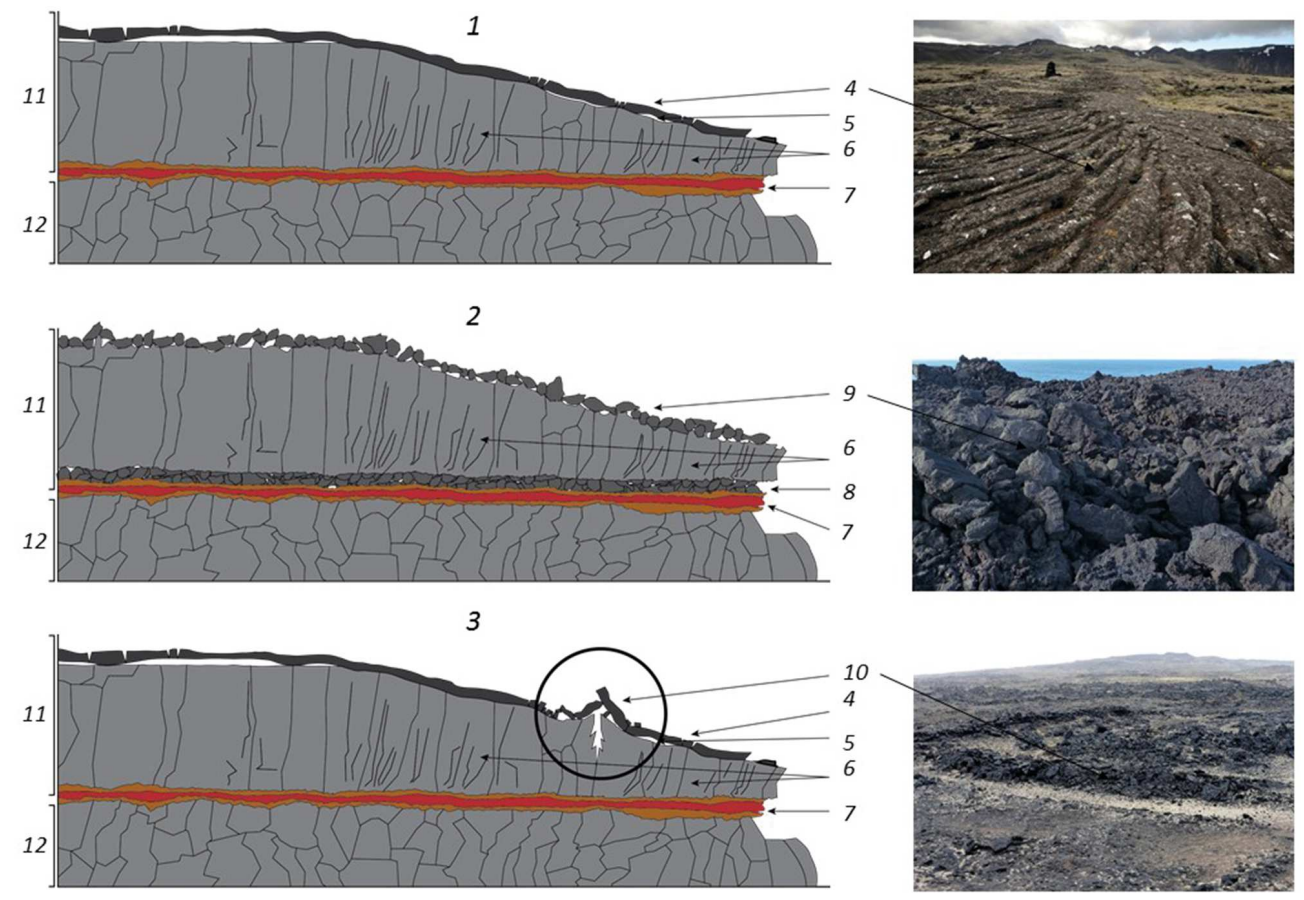

Fig. 2 - Schéma conceptuel des différents types de coulées de lave rencontrées sur la péninsule de Reykjanes.

1 : coulée pahoehoe $; 2$ : coulée 'a'a ; 3 : tumulus, failles et rides de pression ; 4 : surface lisse, cordée ou plissée ; 5 : poche d'air ; 6 : cœur de coulée prismé $; 7$ : horizon intercoulée $; 8$ : couche de blocs scoriacés $; 9$ : couche de lave " en blocs » de surface $; 10$ : tumulus, faille ou ride de pression; 11 : coulée affleurante ; 12 : coulée support.
Fig. 2 - Conceptual diagram outlining the different types of lava flows along the Reykjanes peninsula.

1: pahoehoe flow; 2: 'a'a flow; 3: tumuli, cracks and pressure ridges; 4: smooth, hummocky or ropy surface; 5: air pocket; 6: prismatic flow interior; 7: interflow horizon; 8: bottom (clinkery layer); 9: clinkery surface; 10: tumuli, crack or pressure ridge; 11: outcropping flow; 12: standing flow. 
Les épisodes éruptifs qui intéressent les affleurements basaltiques de la zone d'étude sont datés entre 750 et 14500 ans BP (fig. 1; Sigurgeirson, 1995).

Lâge de ces coulées assure que les blocs rencontrés sur les platesformes sommitales de ces falaises ne correspondent pas à des blocs erratiques puisque leur mise en place est postérieure à la dernière glaciation (MIS 2-4 et 5a-d). Il est également possible déliminer toute origine glacielle, car la côte sud-ouest de l'Islande a été épargnée de la formation d'une banquise, notamment du fait de l'advection d'eau atlantique chaude par le courant d'Irminger (Andrews, 2005). Les calottes glaciaires les plus proches étant situées $150 \mathrm{~km}$ plus à l'est, la région est hors de portée des puissantes débâcles fluvioglaciaires que sont les jökulhlaups (Russell et al., 2005). Enfin, n'ayant aucune information historique ou holocène attestant de l'existence de tsunami à léchelle du bassin nord-Atlantique, nous restons réservés sur cette possibilité en matière de processus de mise en place de ces dépôts. Les seuls travaux indiquant des dynamiques liées à l'action de tsunami concernent les études réalisées sur l'effet des glissements sous-marins affectant les pentes occidentales de la plate-forme continentale norvégienne "Storegga Tsunami ", notamment l'épisode daté aux environs de $8100 \mathrm{BP}$ qui a affecté le nord-ouest de la Norvège (Bondevik et al., 1997) et les îles Shetland (Bondevik et al., 2005), et plus tardivement celui daté de $7100 \mathrm{BP}$ qui a touché le littoral oriental écossais (Dawson et al., 1988 ; Long et al., 1989).

Les différents secteurs où l'on trouve des accumulations de CTSD présentent des contextes lithostructuraux différents. En effet, certains sites se trouvent sur des coulées lisses de type pahoehoe et d'autres sur des coulées à la topographie chaotique de type 'áa (fig. 2). Les coulées pahoehoe sont issues de lécoulement d'une lave fluide sur un terrain peu accidenté (Peterson et Tilling, 1980). La couche de surface est harmonieuse et prend souvent l'aspect de cordes plissées. La perte de gaz volcaniques, la baisse dela température, leschangements de topographie du terrain découlement et l'augmentation de la
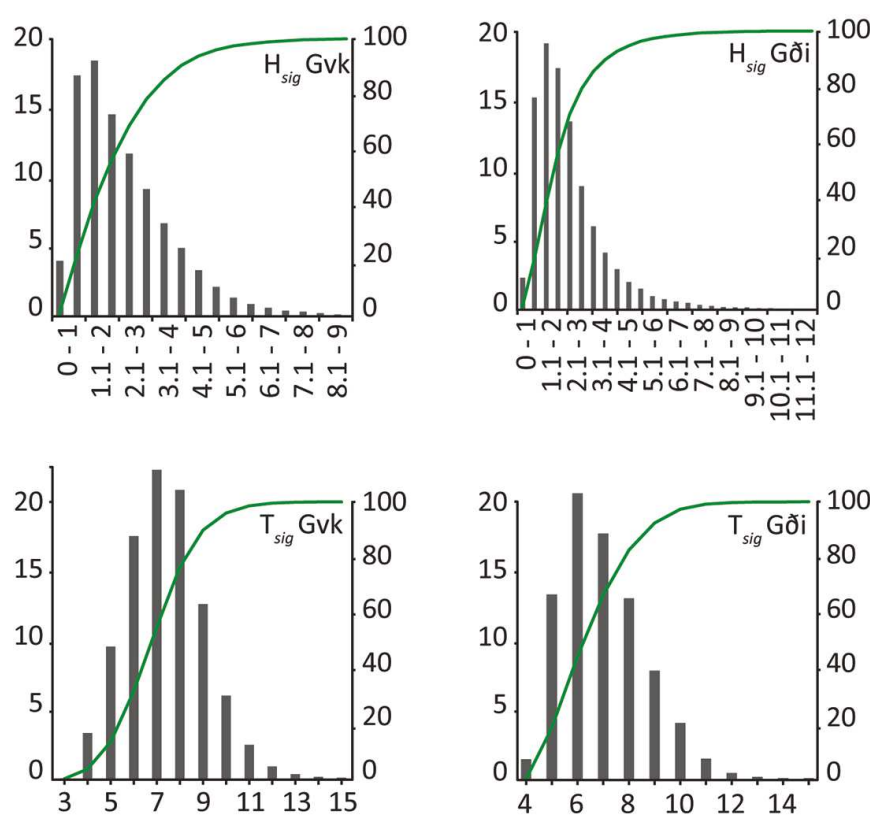

Fig. 3 - Histogrammes des hauteurs significatives de la houle (Hsig) et des périodes significatives de la houle (Tsig) mesurée par les bouées Grindavík (Gvk) et Garðskagi (Gði) (moyenne sur 10 ans, entre janvier 1994 et avril 2004).

Fig. 3 - Histograms of significant wave heights (Hsig) and significant wave periods (Tsig) based on data obtained by measurements (Grindavík buoy (Gvk) and Garðskagi buoy (Gði)) between 1994, January and 2004, April. viscosité des coulées pahoehoe au cours de leur écoulement sont des facteurs susceptibles de les transformer en coulées 'àa. Ces dernières sont le produit de l'écoulement d'une lave plus visqueuse. La couche de surface, dont la solidification est plus rapide, est fragmentée par la remontée des gaz volcaniques en provenance du cœur et par l'effet de l'écoulement interne plus rapide. Ce phénomène confère aux surfaces des coulées 'áa une forme chaotique, recouvertes de scories et de blocs rugueux. Pour ces deux types de coulées, le cœur est dense et massif, et repose sur un niveau scoriacé (semelle) qui est d'ordre décimétrique pour les coulées pahoehoe et métrique dans le cas des coulées 'a’a.

La succession de couches de laves construit des empilements puissants marqués par un réseau de diaclases très denses et hétérogènes, dans lesquels sont taillées les falaises que nous avons étudiées. De manière générale, le cœur massif des coulées est découpé en forme de prismes verticaux réguliers. Cette fissuration dorigine structurale est liée aux conditions de refroidissement des coulées de lave. Localement, le volcano-tectonisme particulièrement actif de la région est responsable de la mise en place de réseaux de failles (Gudmundsson, 1987). Elles affectent le basalte plus en profondeur selon un axe sud-ouest/nord-est. La pression et la libération de gaz volcaniques au cours du refroidissement de la lave créent également des zones de fragilité abondantes à la surface des coulées de la péninsule de Reykjanes, telles que les rides de pression et les tumulus (fig. 2).

\subsection{Conditions hydrodynamiques}

Lanalyse hydrodynamique repose sur des données de houle mesurées par la bouée Grindavík située à 1,6 mille nautique de la côte $\left(63^{\circ} 48,80^{\prime} \mathrm{N} ; 22^{\circ} 27,63^{\prime} \mathrm{E}\right.$; prof. $\left.62 \mathrm{~m}\right)$, et Garðskagi située à 7 milles nautiques $\left(64^{\circ} 3,150^{\prime} \mathrm{N} ; 22^{\circ} 52,62^{\prime} \mathrm{E}\right.$; prof. $60 \mathrm{~m}$; fig. 1$)$.

La zone d'étude est soumise à une hydrodynamique très énergétique en raison de son exposition aux houles de tempêtes générées par les dépressions nord-atlantiques non amorties par la bathymétrie plongeante de l'avant-côte (fig. 1). Les falaises basaltiques de la zone étudiée ont une exposition mixte aux houles formées en Atlantique Nord par les systèmes dépressionnaires. Sans tenir compte des effets de diffraction autour des pointes de Kerling et de Reykjanestá (fig. 1), la moitié du linéaire côtier étudié est soumis à laction des houles de secteur ouest / sud-ouest, l'autre moitié étant sous l'influence dominante des houles de sud à sud-est. Au cours dévènements tempétueux, les hauteurs significatives de la houle mesurées aux bouées Garðskagi et Grindavík peuvent dépasser les $17 \mathrm{~m}$. Cependant, la fréquence d’occurrence de ces hauteurs significatives (Hs) extrêmes est faible $(\mathrm{Hs} 98 \%=7,2 \mathrm{~m}$; Hslan = 12,2 $\mathrm{m}$; Hs100ans = 17,4 m ; fig. 3). La bathymétrie, peu complexe, est caractérisée par une plongée rapide des isobathes le long d'un talus côtier, atteignant $50 \mathrm{~m}$ de fond à moins d'un kilomètre des côtes (fig. 1). Cette morphologie, dans un contexte où le marnage maximum atteint $4 \mathrm{~m}$, évite tout phénomène de dissipation de lénergie de la houle au large, rendant la côte particulièrement énergétique, depuis le proche infratidal jusquà la zone supratidale.

\section{Matériel et méthodes}

Les mesures réalisées sur le terrain d’avril à juin 2014 ont permis d'obtenir des paramètres quantitatifs dans le but délaborer la typologie des dépôts de blocs supratidaux de la péninsule de Reykjanes. Ils concernent essentiellement les caractéristiques morphologiques des sites et les dimensions des blocs constituant les amas. À cela s'ajoute l'analyse des modalités d'arrachement, de transport et dépôt des mégablocs. Ces dernières ont été caractérisées à partir de létude de 
photos diachroniques obliques obtenues lors de précédentes missions sur le terrain $(2007,2008,2010$ et 2014). Elles permettent de distinguer les secteurs où les changements morphosédimentaires affectant les amas de blocs ont été (et sont) les plus importants, et donnent par là même quelques éléments sur le caractère hydrodynamique de chacun des sites étudiés.

\subsection{Mesures topomorphologiques}

Dans le cadre de cette étude, 35 accumulations supratidales (barrages, amas ou blocs isolés) ont fait l'objet de mesures topomorphosédimentaires (fig. 4). 89 profils transversaux ont été réalisés perpendiculairement ou au droit des accumulations de manière à reconstituer la morphologie de la falaise et des accumulations de blocs situées à son sommet (fig. 4). Les mesures topographiques ont été faites à l'aide d'un GPS Trimble Geo-Explorer XH 6000 dont la précision après post-traitement est de lordre de 5 à $10 \mathrm{~cm}$. Les positions altitudinales du pied des escarpements les plus hauts ont été relevées quant à elles à l'aide d'un topomètre laser TruePulse 360B (précision $\pm 0,5 \mathrm{~m}$ ) du fait de conditions d'accessibilité difficiles ou dangereuses.

\subsection{Taille et émoussé des blocs}

Pour chaque accumulation, la taille des 5 plus gros blocs a été mesurée à partir des axes « $\mathrm{a}$ » (axe le plus long), « $\mathrm{b}$ » (axe intermédiaire) et

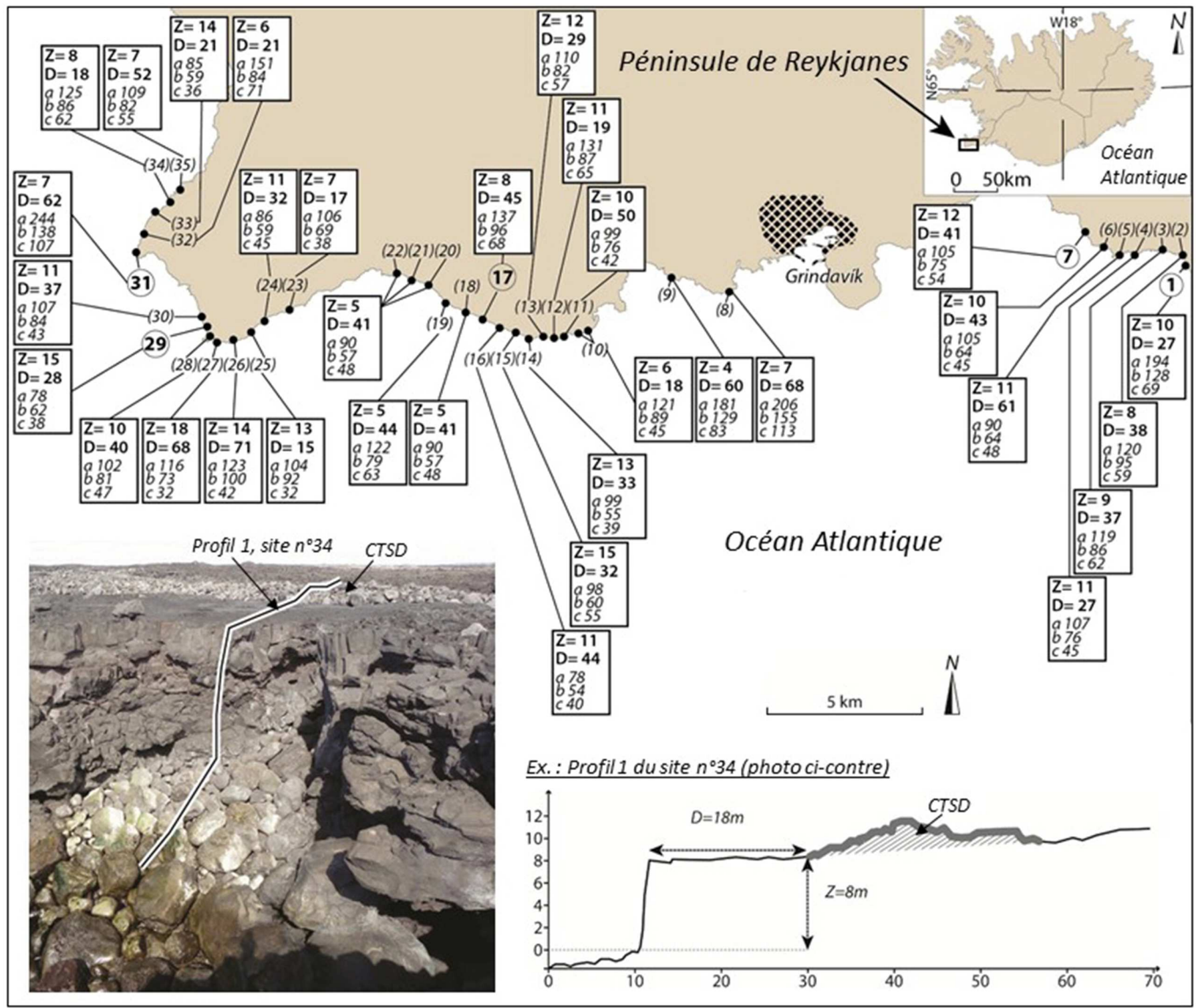

Fig. 4 - Altitude (Z), distance (D) et axes « a ", " b " et « $c$ » (5 plus gros blocs/profil) moyennés des 35 dépôts de blocs supratidaux de sommet de falaise ayant fait l'objet de mesures topo-morphosédimentaires.

Noms des sites : 1 : Selatangar ; 2 : Moshólar ; 3 :Katlahraun ; 4 : Hraunsnes est ; 5 : Hraunsnes ouest ; 6 : Skollahraun ; 7 : Rangargjögur ; 8 : Stekkjarhóll ; 9 : Markhóll ; 10 : Staðarmalir ; 11 : Berghraun ; 12 : Klaufir 1; 13 : Klaufir $2 ; 14$ : Klaufir $3 ; 15$ : Klaufir 4 ; 16 : Klaufir 5 ; 17 : Lynghólshraun ; 18 : Sölvabás ; 19 : Staðarberg ; 20 : Hróabás 21:Brimketill;22:Mölvík;23:Kriki est;24:Krikiouest;25:Skemmur;26:Skarfasetur; 27 : Reykjanestá lighthouse ; 28 : Reykjanestá ; 29: Valbjargagjá sud ; 30 : Valbjargagjá nord ; $31:$ Kerling; 32 : Hak; $33:$ Kinnaberg ; 34 : Eldborg sud ; 35 : Eldborg nord.
Fig. 4 - Mean altitude (Z), distance (D) and " $a$ ", " $b$ ", " $c$ " axis (5 largest boulders on each profile) of the 35 cliff-top storm deposits surveyed by GPS.

Sites: 1: Selatangar; 2: Moshólar; 3:Katlahraun; 4: Hraunsnes est; 5: Hraunsnes ouest; 6 : Skollahraun; :Rangargjögur; 8:Stekkjarhóll;9:Markhóll;10:Staðarmalir;11:Berghraun; 12: Klaufir 1; 13: Klaufir 2; 14: Klaufir 3; 15: Klaufir 4; 16: Klaufir 5; 17: Lynghólshraun 18: Sölvabás; 19: Staðarberg; 20: Hróabás; 21: Brimketill; 22: Mölvík; 23: Kriki, east; 24 Kriki, west; 25: Skemmur; 26: Skarfasetur; 27: Reykjanestá lighthouse; 28: Reykjanestá; 29: Valbjargagjá, south; 30: Valbjargagjá, north; 31: Kerling; 32: Hak; 33: Kinnaberg; 34: Eldborg, south; 35: Eldborg, north. 
A14

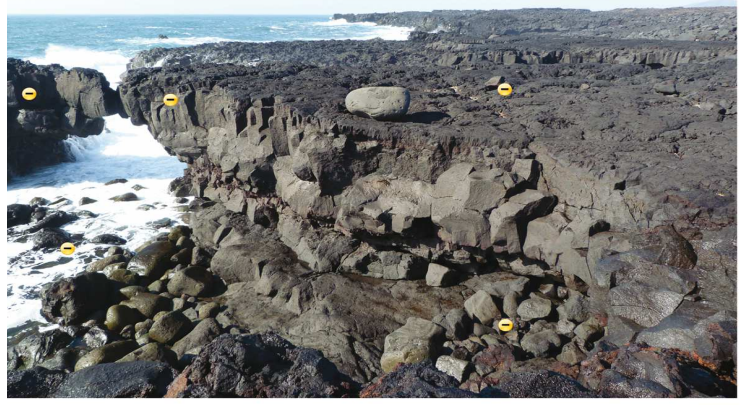

B14
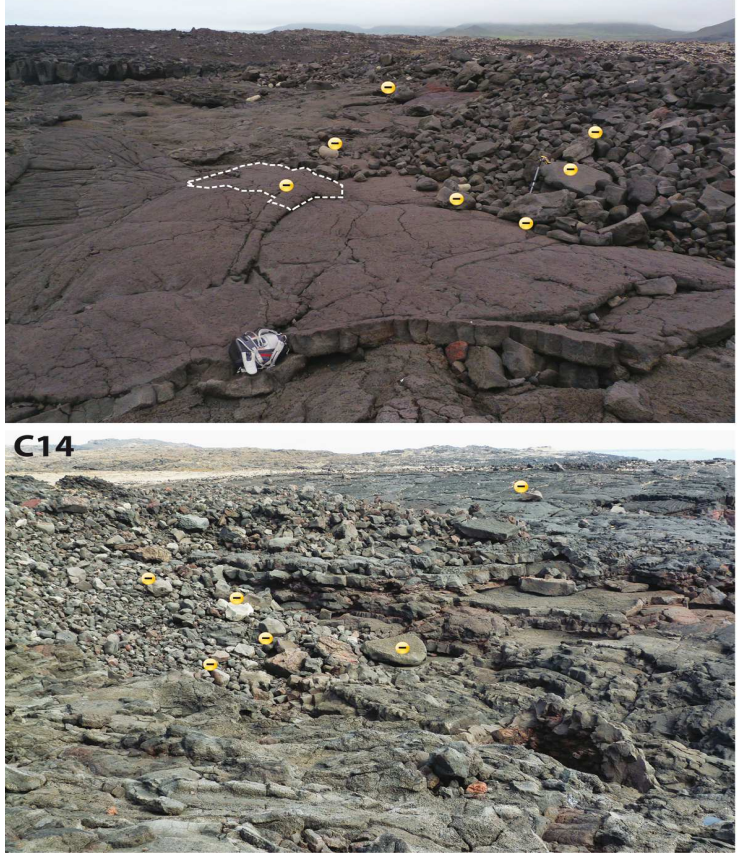

Fig. 5 - Extraits du jeu de données des photos diachroniques obliques illustrant les modifications significatives de la morphologie du trait de côte et des plages de blocs (A), des plates-formes sommitales (B) et des amas de blocs de sommet de falaises (B, C).

« $\mathrm{c} »$ (axe le plus court ; fig. 4). Leur forme a été systématiquement décrite telle que quadrangulaire, ovoïde ou polymorphe. Dans le même temps, le degré démoussé a été mesuré pour 100 blocs répartis le long de chaque accumulation, afin d'analyser la distribution morphométrique au sein de ces dernières (arrondis, subarrondis, subanguleux, anguleux). Ces informations ont permis détablir une caractérisation morphosédimentaire de chaque accumulation et de son environnement de dépôt.

\subsection{Analyse diachronique des modalités de transport et de dépôt}

Létude des dynamiques de transport et de dépôt des de blocs a reposé quant à elle sur l'analyse diachronique de photographies obliques prises depuis le sol au cours des dix dernières années (fig. 5). Cette approche méthodologique mise en œuvre dans le cadre détudes similaires sur d'autres sites en Atlantique, notamment l'île de Banneg dans le Finistère (Fichaut et Suanez, 2008, 2011), vient pallier l'absence de relevés qui sont généralement effectués à partir de plates-formes aériennes. Elle a permis d'apprécier l'importance des changements morphosédimentaires survenus après les hivers les plus tempétueux,
A15

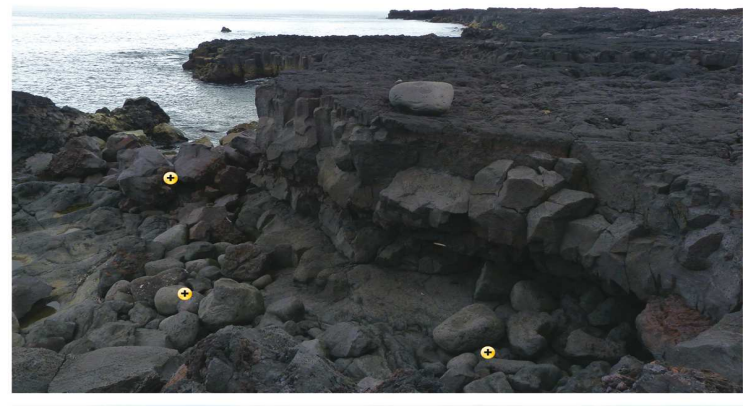

B15
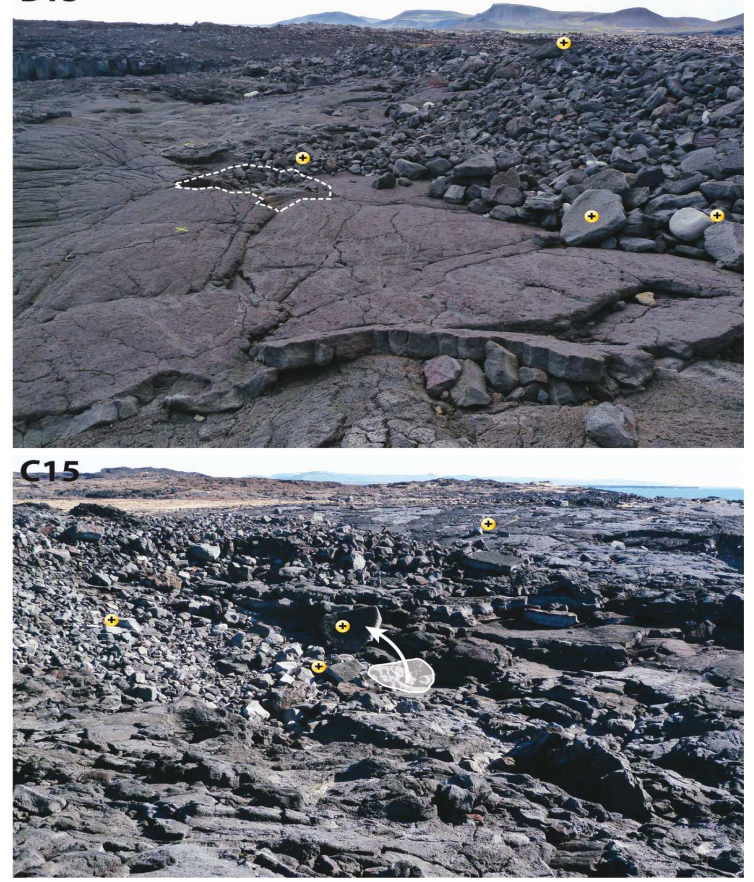

Fig. 5 - Extracts from the oblique photograph dataset showing the morphological changes of the rocky coast $(A)$, boulder beaches $(A)$, wavescoured platforms $(B)$ and cliff-top boulder ridges or clusters $(B, C)$.

et d'identifier les secteurs les plus dynamiques.

\section{Résultats}

La typologie présentée ici (fig. 6) découle d'une analyse multicritères intégrant des paramètres qui sont (i) la structure $\mathrm{du}$ substrat rocheux, (ii) la morphologie des falaises, des estrans quelles dominent et de leurs plates-formes sommitales, (iii) l'hydrodynamisme.

Le substrat rocheux est ici toujours basaltique et sous forme de coulées horizontales à subhorizontales, constituées d'une ou plusieurs unités laviques. Ces ensembles lithologiques sont en général entaillés en falaises verticales dont la hauteur varie de quelques mètres à plus de 15 mètres, en fonction de lépaisseur de la coulée et du nombre de bancs (fig. 6A-C). Ces falaises sont parfois entaillées dans une succession de coulées séparées par une semelle scoriacée qui constitue un niveau de faiblesse favorisant lérosion différentielle (Etienne, 2007) et permettant le développement de plates-formes d'érosion marine ou de falaises à ressauts multiples (fig. 6D1-D2). La structure de la partie superficielle des coulées varie selon qu'elles sont de type pahoehoe ou 'áa. Toutes choses égales par ailleurs, 
(A)

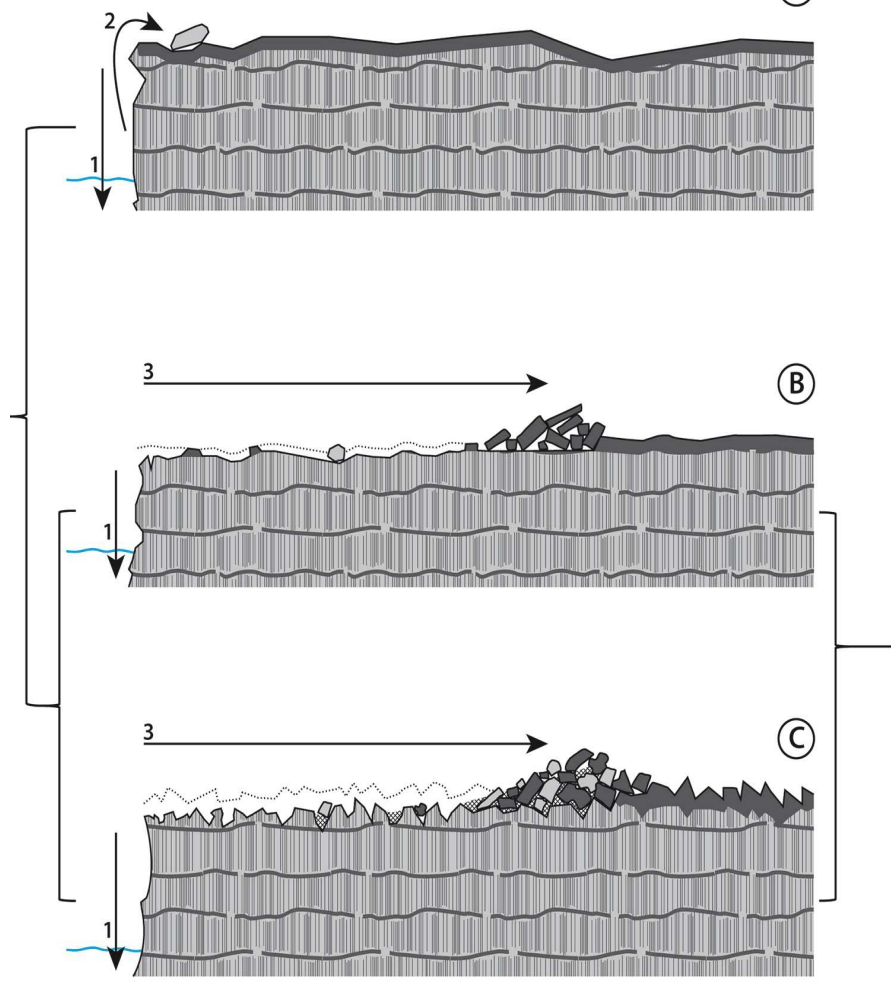

Fig. 6 - Typologie des dépôts de blocs de sommet de falaise de la péninsule de Reykjanes.

A : bloc isolé sur le sommet de la falaise ; B : guirlande de dépavage des surfaces pahoehoe ; C : barrage issu du déblaiement des surfaces 'a'a ; D.1 : amas de boules en retrait de falaises dominant une plate-forme d'érosion marine; D. 2 : amas de boules en retrait des falaises à ressauts multiples; $\mathrm{E}$ : barrage à mégablocs; $1:$ chute de pierres ; 2 : projection sur le sommet de la falaise ; 3 : transit transversal ; 4 : basalte ; 5 : horizon superficiel ; 6 : blocs ; 7 : niveau des hautes mers de vive-eau.

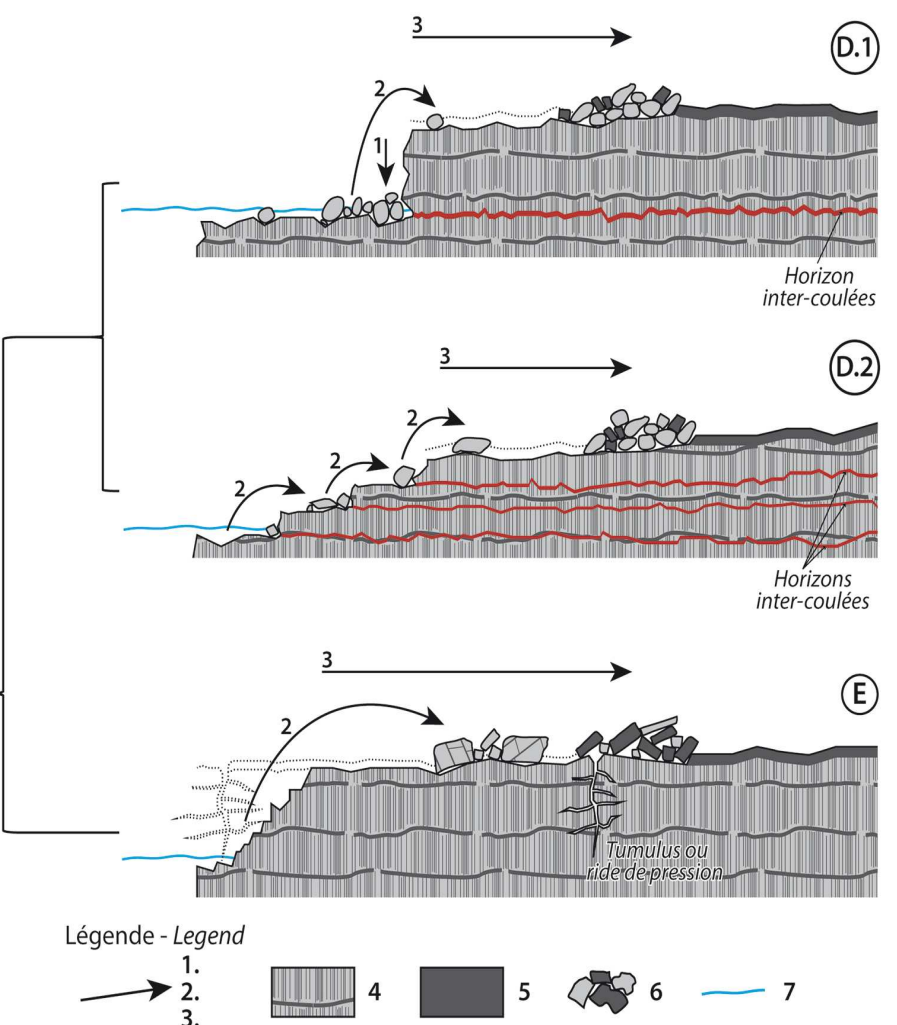

Fig. 6 - Conceptual diagram of the typology of the cliff-top storm deposits of the Reykjanes Peninsula.

A: isolated cliff-top boulder; B: garland-shaped scour in pahoehoe flows; C: ridges of material derived from 'a'a surfaces; D.1: cluster of rounded boulders overhanging a wave-cut platform; D.2: cluster of rounded boulders behind a cliff cut in several intermediate platforms; E: megaclast ridges; 1 : rock fall; 2: boulder projection; 3: cross-shore transit; 4: basalt; 5: superficial horizon; 6: boulders; 7: astronomical high tide level.
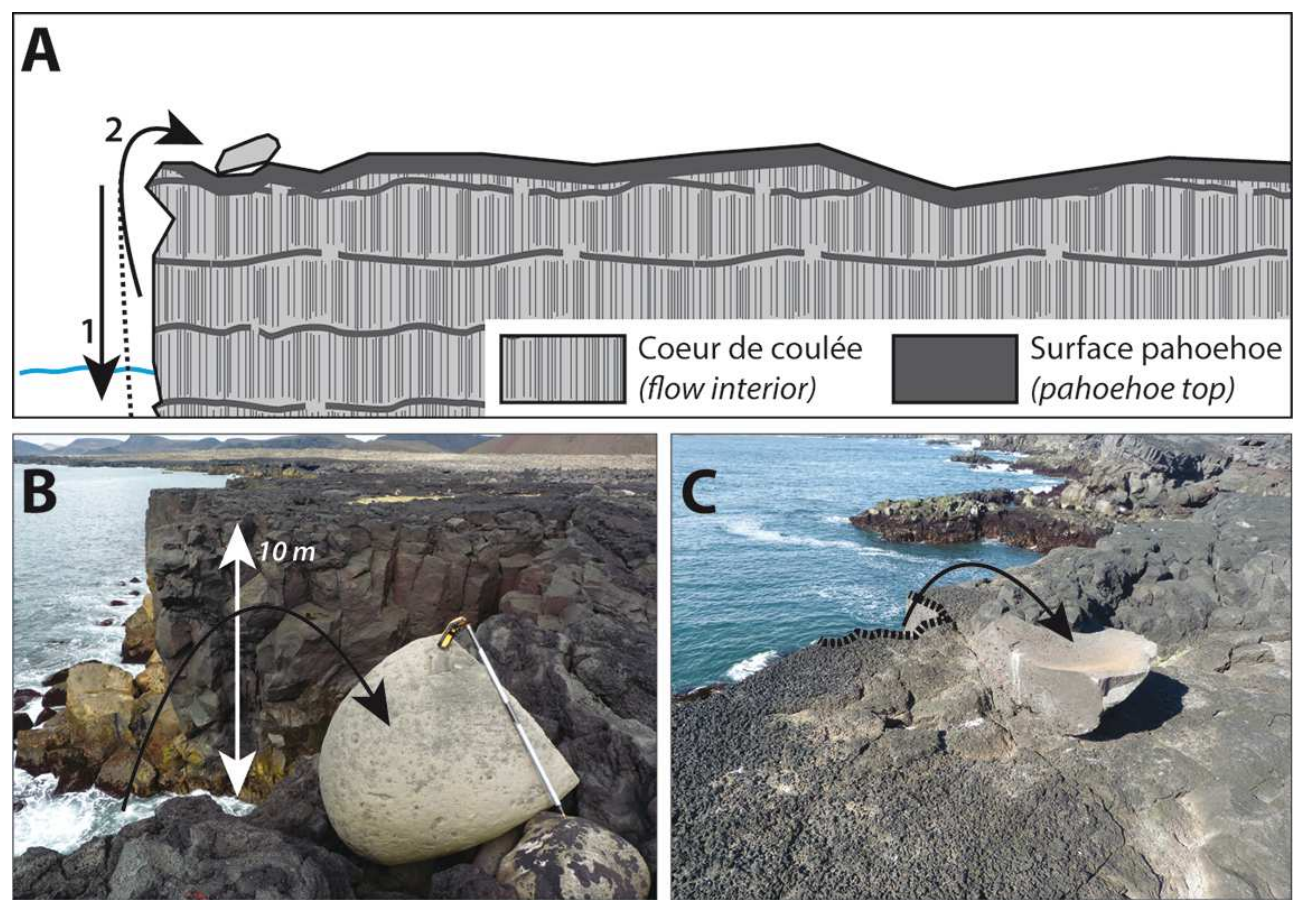

Fig. 7 - Blocs isolés sur le sommet de la falaise.

A : schéma conceptuel basé sur de la mesure topomorphologique $: 1:$ chute de pierres ; 2 : projection sur le sommet de la falaise ; $\mathrm{B}$ : bloc arrondi en provenance de la plateforme d'érosion marine ; $\mathrm{C}$ : bloc anguleux arraché à la falaise (cicatrice en pointillé).
Fig. 7 - Isolated cliff-top boulders.

A: Conceptual diagram based on measurements; 1 : rock fall; 2: boulder projection $B$ : rounded boulder from the wave-cut platform; C: angular boulder from the cliff edge (scar in dotted line). 
les amas sont de taille relativement réduite et séparés du rebord de falaise par une plate-forme peu accidentée, voire lisse pour les premières (fig. 6A). Dans le cas de coulées 'áa, les amas sont plus volumineux et reposent en arrière d'une plate-forme à topographie extrêmement chaotique (fig. 6B). Si les houles enregistrées au large du secteur détude (fig. 1,3) montrent un contexte hydrodynamique particulièrement énergétique, celui-ci présente toutefois des variations locales qui sont fonction de l'exposition entre saillants et rentrants, et de la topographie de l'estran et des falaises. Ce contexte permet, entre

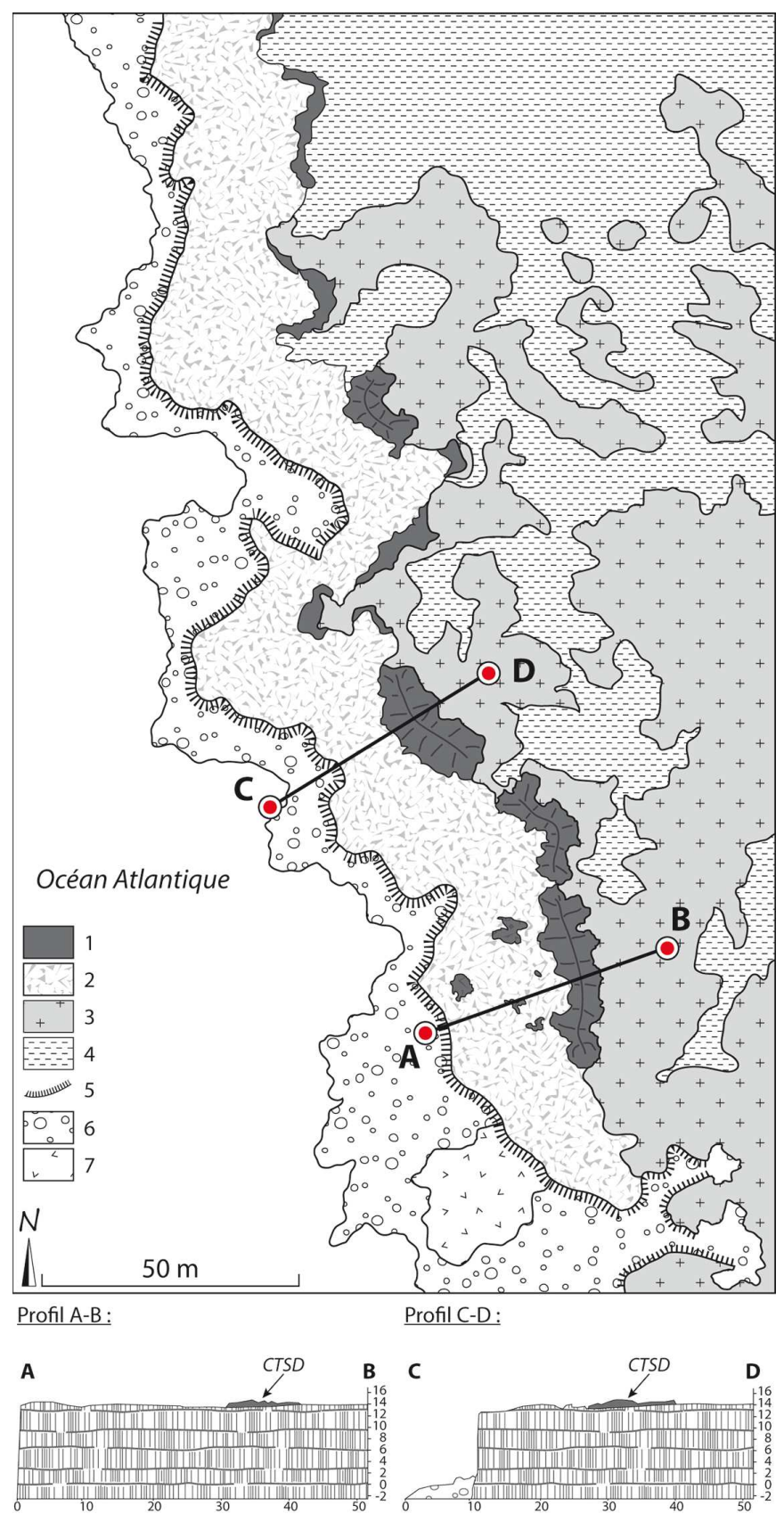

Fig 8 - Carte géomorphologique simplifiée de la guirlande de dépavage de Valbargagja et profils associés.

1 : guirlande de blocs ; 2 : plate-forme sommitale ; 3 : roche en place ; 4 : tephras ; 5 : falaise ; 6 : plage de blocs; 7 : plate-forme d'érosion marine.

Fig 8 - Simplified geomorphological map of the cliff-top boulder garland of Valbargagja, and associated profiles.

1: cliff-top boulder garland; 2: wave-scoured platform; 3: bedrock; 4: tephra; 5: cliff; 6: boulder beach; 7: wave-cut platform. autres, d'expliquer l'existence de barrages comprenant des mégablocs parfois localisés très en arrière du bord des falaises (fig. 6E).

\subsection{Les blocs isolés}

Des blocs isolés sont localement positionnés sur le rebord des falaises les plus hautes (fig. 7A). La mise en place de ces éléments est issue de processus synchrones lorsque la projection du bloc sur la plate-forme sommitale suit immédiatement son arrachage à la paroi de la falaise. Dans ce cas, les blocs sont anguleux à subanguleux selon la fréquence de leur remaniement à la suite de leur dépôt sur le sommet de la falaise (fig. 7C). La présence de blocs émoussés, voire de boules, résulte de processus successifs lorsque les blocs ont été dans un premier temps détachés de l'abrupt de la falaise, sont tombés dans la zone intertidale sur la plate-forme dérosion marine où ils ont acquis leur forme caractéristique, avant d’être projetés sur la plateforme sommitale (fig. 7B). Au regard du volume que représentent les types de dépôts qui sont introduits ci-après, les blocs isolés ne concernent qu'une très faible proportion des dépôts observés.

\subsection{Les guirlandes de dépavage des coulées pahoehoe}

Ce type de dépôt est appelé ainsi en raison de sa forme de cordon sinueux et discontinu à lallure d'une guirlande, dont la crête sommitale est grossièrement parallèle à la ligne du bord de la falaise à plusieurs dizaines de mètres en arrière (fig. 8). Ces accumulations de blocs sont issues du dépavage des coulées pahoehoe (Etienne, 1997) et sont imputables aux processus de météorisation mécanique qui affectent ces surfaces : écaillage en « croûte de pain » (Etienne et André, 2003) ou action mécanique des masses d'eau issues du déferlement retombant sur le sommet de falaise (fig. 9A). Le débitage des petits blocs se fait le long de zones de faiblesses structurales apparues lors de la solidification puis exploitées par la météorisation ou par la force hydrodynamique des paquets de mer. Un détachement lié à l'action du gel par effet de coin affecte les surfaces vacuolaires et favorise la désolidarisation des pavés de la coulée support. La gélidivision des couches superficielles fournit alors un matériel sous forme d'une couverture de blocs décimétriques. Les paquets de mer qui retombent sur le sommet de falaise exercent, quant à eux, une action de disjonction due au coup de bélier provoqué par la masse d'eau (Sunamura, 1988).

Le dépavage de cette croûte se fait donc lors du passage d'une nappe d'eau turbulente ou lors du déferlement des vagues sur la surface météorisée. Cette interprétation suggère que le sommet des falaises puisse être submergé lors des épisodes morphogènes, comme cela a pu être observé sur lîle de Banneg en Bretagne (Sheremet et al., 2014). Il en résulte des accumulations de blocs à granulométrie bimodale. $60 \%$ à $80 \%$ du matériel sont constitués de petits blocs issus de la gélidivision $(0,25<$ axe $\mathrm{b}<0,5 \mathrm{~m})$, tandis de $20 \%$ à $40 \%$ du matériel restant sont des blocs moyens et/ou des blocs grossiers $(0,5<$ axe $b<2 \mathrm{~m})$. Ces derniers, qui ont une forme de dalles, sont le résultat du décapage par les vagues de surfaces non gélidivisées. En général, le cœur de coulée en colonnades présente peu de faiblesses structurales en dehors des joints prismatiques ; il est alors épargné du débitage et constitue une plate-forme relativement peu accidentée entre le sommet de falaise et la guirlande située en arrière (fig. 9A1-2). Néanmoins, quelques blocs plus grossiers dont la forme prismatique témoigne de leur arrachage au cœur de la coulée ont également été inventoriés dans ce type d'accumulation.

La position de la zone d'accumulation des blocs est fonction de la topographie. Les barrages qui reproduisent grossièrement le tracé du front de falaise peuvent être proches de celui-ci si le matériel en transit est bloqué par un obstacle topographique. Ils peuvent aussi se situer en position plus ou moins éloignée si aucun saillant ne vient arrêter 

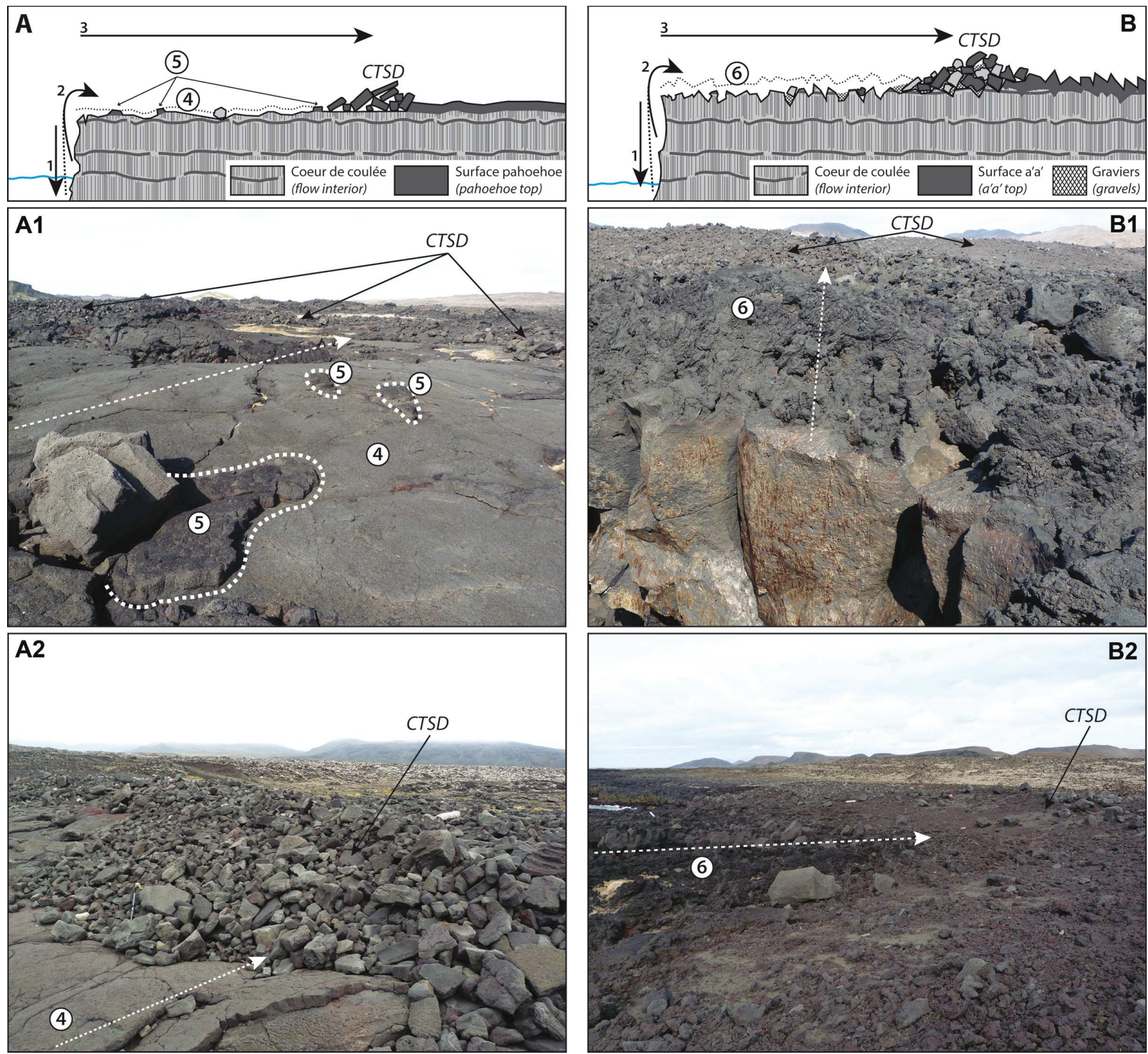

Fig 9 - Guirlandes de dépavage des surfaces pahoehoe (A) ou barrages issus du déblaiement des surfaces 'a'a (B).

A et $B$ : schémas conceptuels basés sur de la mesure topomorphologique ; 1 : chute de pierres ; 2 : projection sur le sommet de la falaise ; 3 : transit transversal ; 4 : plate-forme sommitale de coulée pahoehoe ; 5 : résidus de la " croûte de pain »; 6 : plate-forme sommitale de coulée 'a'a; A1, A2, B1 et B2 : photographies illustratives.

le déplacement des blocs vers l'intérieur. Dans ce cas, seules la pente et la compétence des vagues peuvent jouer comme facteurs limitant la progression des blocs vers l'intérieur des terres.

\subsection{Les barrages de déblaiement superficiel des coulées 'a’a}

Comme pour le dépavage des coulées pahoehoe, le déblaiement des coulées 'áa est un processus qui agit à leur surface (fig. 9B). Il est aussi la conséquence du passage d'une nappe d'eau véloce ou du déferlement des vagues au-delà du sommet de la falaise. La couche de scories et de blocs qui se trouve en surface est alors entièrement remaniée et déblayée. Cette action met à nu une surface dérosion très accidentée (fig. 9B1-B2) et construit des accumulations plus volumineuses
Fig 9 -Garland-shaped scour in pahoehoe flows (A) or ridges of materials derived from 'áa flows $(B)$.

$A$ and B: Conceptual diagrams based on measurements; 1: rock fall; 2: boulder projection; 3: cross-shore transit; 4: wave-scoured platform; 5: residual pahoehoe-top; 6: chaotic wave-scoured platform; A1, A2, B1 and B2: illustrative photographs.

que celles observées sur les coulées pahoehoe. La distance entre les barrages de ce type et le bord de la falaise est très variable. Du fait de la rugosité importante de la plate-forme sommitale, de nombreux blocs de toutes tailles sont localisés en avant du barrage, piégés dans des anfractuosités. De ce fait, il n'existe pas de lien causal entre l'altitude, la distance et la taille des blocs accumulés.

Le matériel présente une granulométrie très hétérogène, allant des graviers aux blocs très volumineux avec une proportion de scories et de grattons importante. Ce dépôt constitue une matrice venant combler les vides interstitiels entre les blocs plus grossiers. Parmi ces derniers, une grande majorité est issue de la couche chaotique qu'ils formaient à la surface de la coulée 'a’a. Le cœur de ces coulées étant plus résistant que celui des coulées pahoehoe (Marie, 2004), peu de 

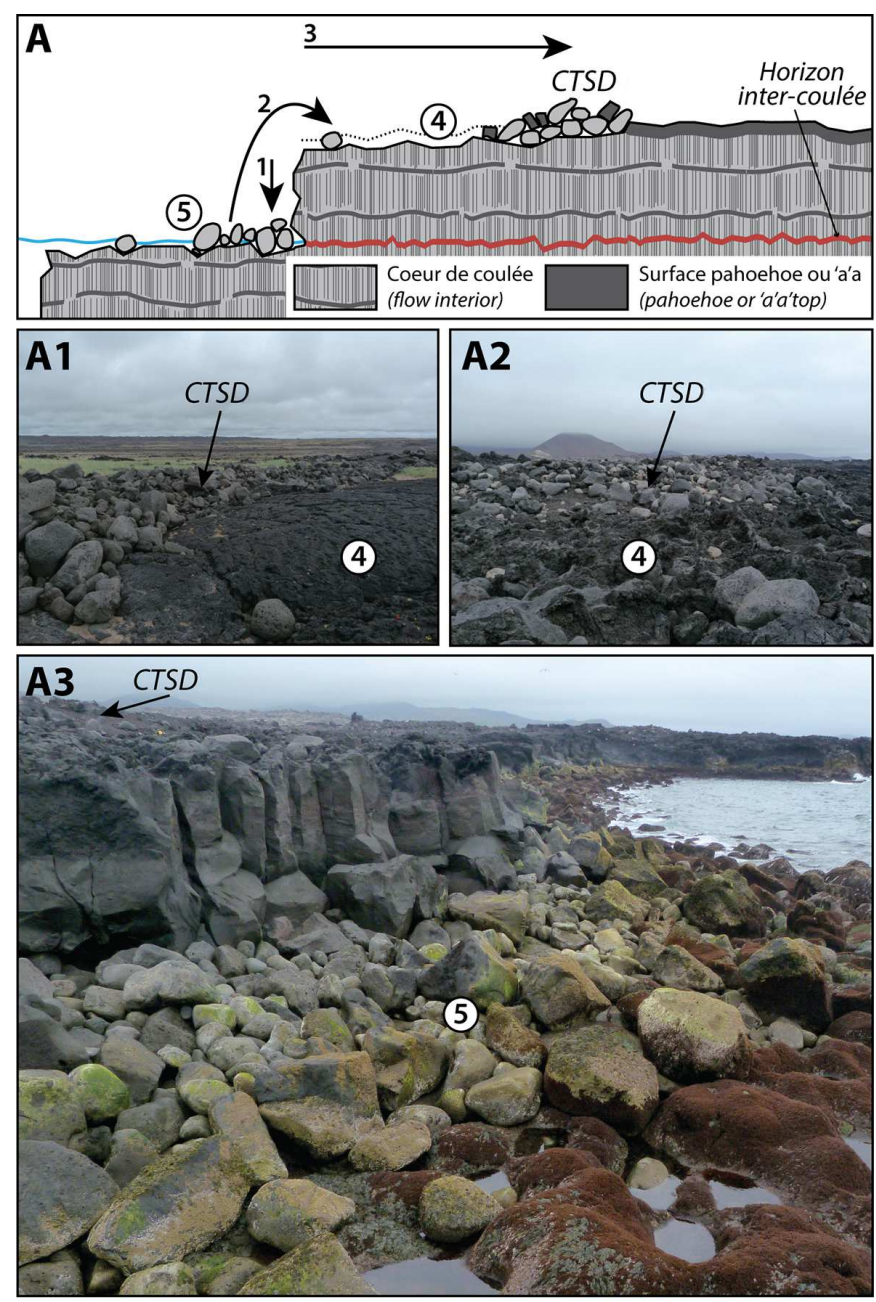

Fig 10 - Les amas de " boules " en retrait de falaises dominant une plateforme d'érosion marine $(\mathrm{A})$ ou en retrait de falaises à ressauts multiples $(\mathrm{B})$. A et $B$ : schémas conceptuels basés sur de la mesure topomorphologique ; $1:$ chute de pierres ; 2 : projection sur le sommet de la falaise ; 3 : transit transversal ; $4:$ surface pahoehoe décapée ; 5 : plage de blocs sur la plate-forme d'érosion marine ; $6:$ platesforme intermédiaires; A1, A2, A3 et B2 : photographies illustratives.

blocs y sont prélevés par les vagues.

\subsection{Les amas de «boules» en retrait de falaises dominant une plate-forme d'érosion marine}

La présence de blocs arrondis à hauteur de $40 \%$ à $60 \%$ dans certaines accumulations montre que d'autres processus interviennent dans la construction des barrages de CTSD (fig. 10A). Très émoussé, ce matériel a nécessairement séjourné dans un premier temps dans la zone intertidale (fig. 10A3) avant dêtre projeté en sommet de falaise et soustrait à l'abrasion marine (fig. 10A1-A2). Le plus souvent, il provient de la plate-forme dérosion marine formée par lérosion différentielle de la falaise, le plan d'entaille étant guidé par les horizons inter-coulées soumis à l’action des vagues (Etienne, 2007). Les blocs de cœur de coulée sont alors mis en porte à faux et tombent sur la plate-forme dérosion où ils sont remaniés lors des tempêtes. D’après les travaux de Moign et Moign (1970) portant sur l'île de Surtsey en Islande, et de Marie (2004) sur Hawaii, il faut entre une à deux années pour que des blocs basaltiques soient totalement émoussés et prennent lallure de "boules ». À la faveur dévènements de très forte énergie, certaines de ces boules sont projetées sur les plates-formes sommitales, et viennent alimenter les barrages de blocs situés plus en arrière.
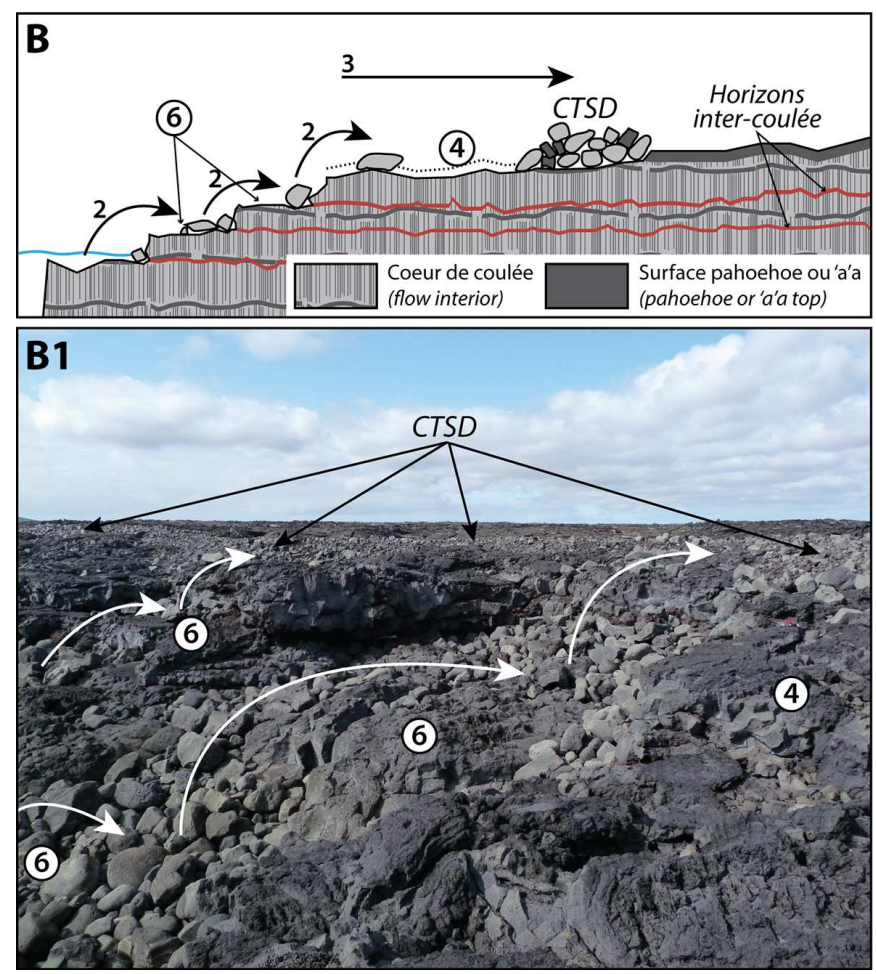

Fig 10 - Clusters of rounded boulders overhanging a wave-cut platform (A) or facing cliff cut in several intermediate platforms $(B)$.

$A$ and B: Conceptual diagrams based on measurements; 1 : rock fall; 2: boulder projection; 3: cross-shore transit zone; 4: wave-scoured platform; 5: wave-cut platform; 6: intermediate platform; A1, A2, A3 and B2: illustrative photographs.

4.5. Les amas de « boules " en retrait de falaises à ressauts multiples

Ce type de dépôt se rencontre sur les sites correspondant aux coulées de lave de Berghraun et de Katlahraun (2-3 ka). Ces derniers présentent des falaises taillées en forme de marches d'escalier. Les sommets des plates-formes intermédiaires correspondent aux toits des bancs de laves qui se sont écoulées lors des éruptions précédentes. Ces sites comportent une fraction plus importante de blocs arrondis, pouvant atteindre $70 \%$ à Berghraun (fig. 1). Leur remontée sur la plate-forme sommitale est facilitée par les escarpements successifs moins hauts (fig. 10B-B1).

\subsection{Les barrages à mégablocs}

Ce type de barrage très volumineux et qui comprend des blocs de très grande taille (fig. 11) n'existe qu'en deux endroits de la côte étudiée. Les deux sites forment les pointes basses saillantes de Kerling et de Reykjanestá qui sétendent vers le large à l'extrême sud-ouest de la péninsule de Reykjanes et sont en conséquence les plus exposés du littoral étudié (fig. 1). D’une part, dans les deux cas, l'isobathe $100 \mathrm{~m}$ est à seulement $2 \mathrm{~km}$ du trait de côte alors que partout ailleurs il est à $3 \mathrm{~km}$ ou plus ; d'autre part, leur position en promontoire avancé 

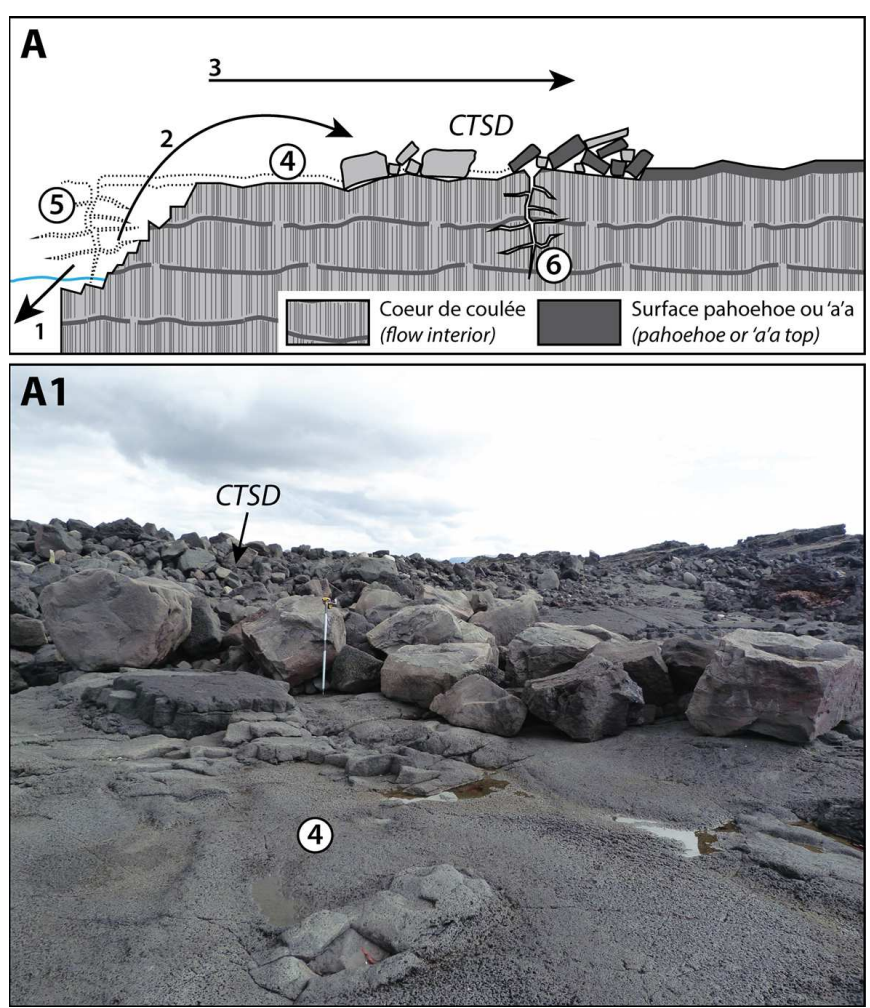

Fig 11 - Barrages à mégablocs.

A : schéma conceptuels basé sur de la mesure topomorphologique ; 1 : chute de pierres ; 2 : projection sur le sommet de la falaise $3:$ transit transversal ; 4 : plate-forme sommitale de coulée pahoehoe ; 5 : tumulus démantelé ; 6 : tumulus; A1 : photographie illustrative.

\section{Fig 11 - Megaclast ridges.}

A: Conceptual diagram based on measurements; 1: rock fall; 2: boulder projection; 3: cross-shore transit zone; 4: wave-scoured platform; 5: degraded tumulus; 6: tumulus; A1: illustrative photograph.

fait qu'ils sont exposés à un large spectre de houles, de nord à sud à Kerling, nord-ouest à est-sud-est à Reykjanestá, c'est à dire aux houles les plus fréquentes et les plus énergétiques (fig. 1). Dans le détail, la configuration des deux sites diffère.

Le barrage de Kerling (fig. 12A), étiré sur 180 mètres selon un axe nord-sud, atteint $60 \mathrm{~m}$ de large et culmine à $10 \mathrm{~m}$. La base de sa face externe est à $6 \mathrm{~m}$ d'altitude et jusquà $80 \mathrm{~m}$ en retrait des basses falaises auxquelles il est grossièrement parallèle. La base du revers du barrage est donc à $140 \mathrm{~m}$ du trait de côte, mais de nombreux blocs épars sont déposés jusquà $200 \mathrm{~m}$ à l'intérieur des terres. Les falaises subverticales ou à ressauts multiples, hautes de 4 à $6 \mathrm{~m}$ environ, sont entaillées dans une coulée à très grands prismes de même épaisseur. En arrière de la falaise et jusqu'à la base du barrage sétend une plateforme extrêmement accidentée mais vierge de tout dépôt (fig. 12A). Dans sa partie médiane elle est en plus traversée par une longue dépression évidée dans une ride de pression dorientation nord nordouest - sud sud-est, dont le fond est localement situé sous le niveau des hautes mers de vives-eaux. Au sud, à $60 \mathrm{~m}$ en arrière du trait de côte, reposent d'énormes blocs. Certains ont été déposés au cours de des tempêtes de l'hiver 2014-2015 (fig. 12B), alors que d'autres en étaient extraits et transportés en direction du barrage où ils s'accumulent sur la face externe selon un gradient de taille décroissant vers le sommet (fig. 12A). La localisation de cicatrices fraîches et le caractère anguleux des blocs montrent que ces derniers proviennent tous du rebord de la falaise et non pas de la plate-forme sommitale elle-même qui n'est qu'une surface de transit.

Ici, de tous les paramètres, c'est la vigueur de l'hydrodynamisme qui explique le caractère exceptionnel de la forme et la présence de mégablocs déposés très en retrait. Étant donné lorientation du barrage, ils résultent de l'action prédominante de houles de secteur ouest peu freinées à l'approche du rivage. Leur déferlement se fait directement sur la falaise ou au-delà, et est prolongé par un jet de rive suffisamment puissant pour empêcher le dépôt de blocs sur une plateforme pourtant très accidentée.

Le barrage de Reykjanestá est un peu plus élevé, puisque sa base est à une altitude comprise entre 8 et $10 \mathrm{~m}$. Lédifice long de $100 \mathrm{~m}$ et large de $30 \mathrm{~m}$ environ, est beaucoup plus proche du bord de la falaise dont il nest séparé que par une plate-forme sommitale horizontale large de 15 à 20 mètres (fig. 13A, profil A-B), sauf vers le sud-ouest où elle est plus étendue, en pente douce et dépourvue de blocs (fig. 13A, profil C-D). D’abord tournée face à l'ouest dans sa partie nord, l'accumulation s'incurve ensuite brutalement pour s'orienter face au sud sud-ouest, ces deux directions étant celles des houles efficaces sur cette pointe extrêmement saillante. Cet ensemble est en fait constitué de deux barrages distincts se raccordant par l'une de leurs extrémités. La pointe est entaillée dans des coulées de lave stratifiées épaisses de plusieurs mètres, montrant un faible pendage vers la mer, et séparées par une semelle de coulée. L'estran, jusqu'au niveau des hautes mers de vives-eaux est une plate-forme correspondant au toit de la coulée inférieure. Il se raccorde brutalement à une falaise verticale, haute de 6 à 8 mètres et entaillée dans la coulée supérieure, qui cerne toute la pointe. Dans les rentrants, au pied de l'abrupt, l'estran est recouvert d'une accumulation de très gros blocs, anguleux lorsqu'ils proviennent d'un effondrement récent de la falaise. Ils sont par contre émoussés, voire arrondis lorsqu'ils sont plus vieux. Loriginalité du barrage de Reykjanestá réside dans la présence de mégablocs, d’une part, mais surtout dans le fait que bon nombre d'entre eux sont des blocs émoussés ou même des boules qui ne peuvent que provenir de l'estran (fig. 13B). Dans un contexte où les falaises hautes de 6 mètres sont partout verticales et non pas taillées en marches descalier ou en rampe plus ou moins inclinées, il est évident que les blocs nont pas pu remonter l'abrupt par à-coups. Ils ont nécessairement été projetés en hauteur et de manière instantanée par des déferlements puissants intervenant directement en pied de falaise dans un contexte paroxysmique. Un autre argument plaide en faveur de cette explication. Il réside dans la très faible largeur (15 à $20 \mathrm{~m}$ ) de la plate-forme sommitale dans un environnement pourtant extrêmement énergétique. Elle ne peut s'expliquer que par un déferlement intervenant directement contre la paroi de la falaise ou à son pied, déferlement suivi non pas d'un jet de rive dans le plan horizontal mais d'un jaillissement puis d'un effondrement subvertical de la masse d'eau directement en arrière de l'obstacle (effet splash).

\section{Discussion et conclusion}

Comme nous l'avons montré dans la partie relative aux guirlandes de dépavage des coulées pahoehoe, la fourniture de matériel sédimentaire sous forme de pavés à la surface des coulées pahoehoe est liée aux conditions de refroidissement de la lave, et leur remaniement est largement subordonné à l'action hydrodynamique des déferlements en sommet de falaises. Ces éléments tendent à minimiser l'action de la gélidivision qui a été pointé par Etienne et André (2003) comme étant le processus de météorisation responsable du dépavage des surfaces pahoehoe. Nous estimons de notre côté qu'il joue un rôle secondaire, et finalement assez négligeable tant dans la fourniture de matériaux alimentant les guirlandes de pavés supratidales que dans l'abaissement des plates-formes sommitales de la frange méridionale de la péninsule de Reykjanes.

L' existence de 2 barrages incluant des mégablocs de masse comprise entre 5 et 25 tonnes pose des questions pour le moment non résolues sur les modalités de transport liées au déferlement de vagues géantes 

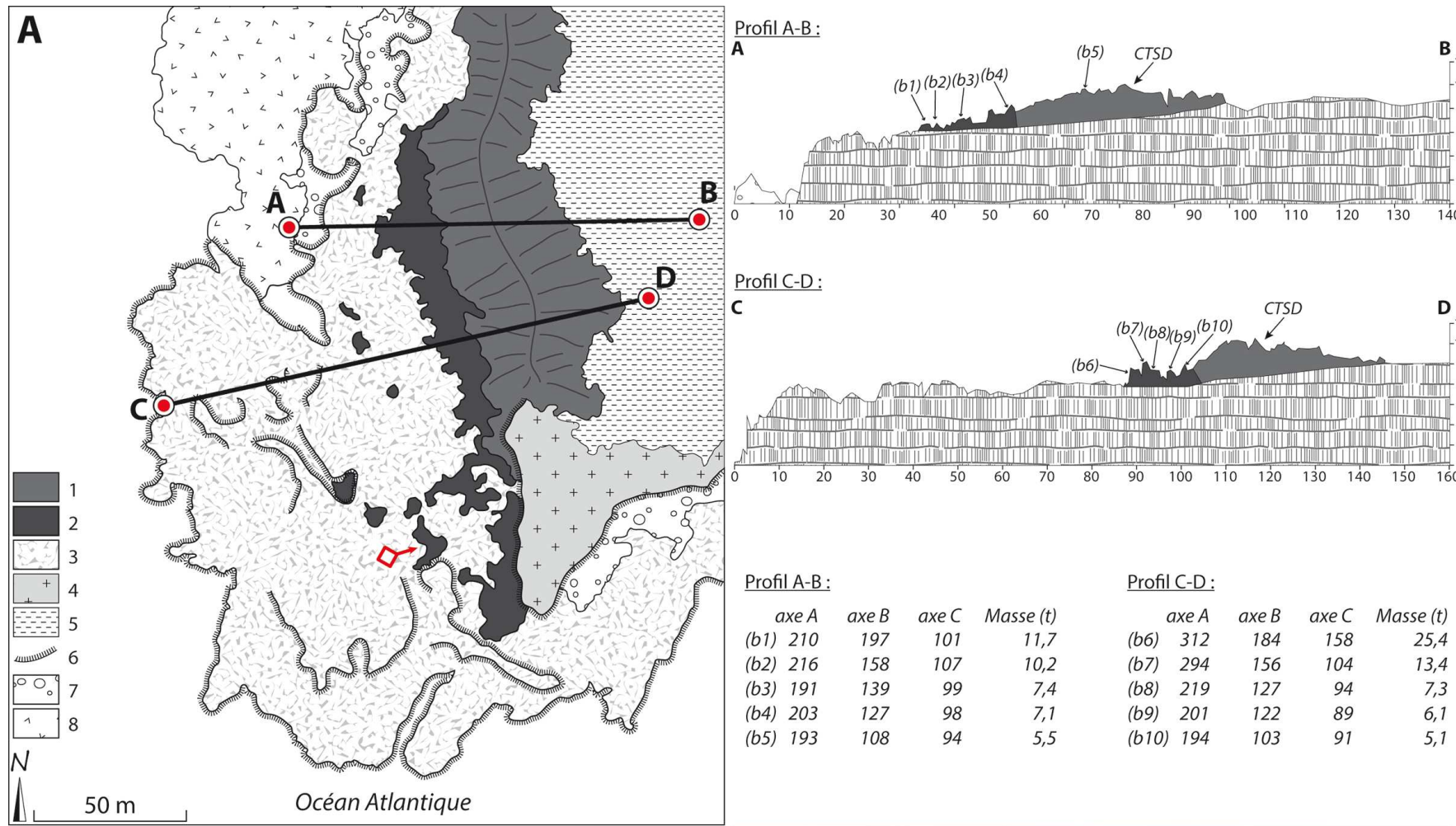

\section{B14}

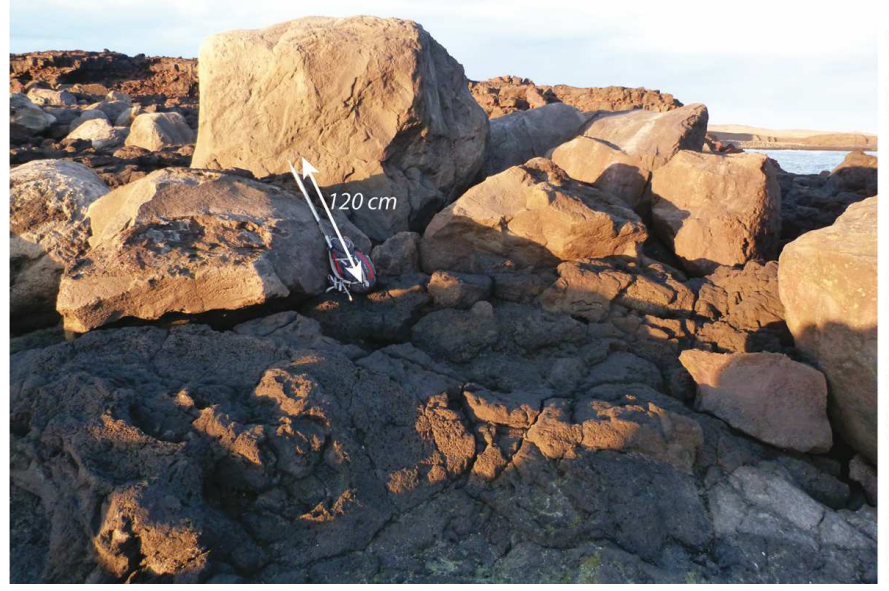

Fig 12 - Carte géomorphologique simplifiée du barrage à mégablocs de Kerling avec les profils associés $(\mathrm{A})$, les mensurations des cinq plus gros blocs et les photographies obliques comparatives entre 2014 (B14) et 2015 (B15).

1 : guirlande de blocs; 2 : mégablocs; 3 : plate-forme sommitale ; 4 : roche en place ; 5 : tephras ; 6 : falaise ; 7 : plage de blocs ; 8 : plate-forme d'érosion marine.

à la côte. Situé à $6 \mathrm{~m}$ d'altitude et à $80 \mathrm{~m}$ du rebord de la falaise, le barrage de Kerling est haut de $4 \mathrm{~m}$ et large de $60 \mathrm{~m}$. En arrière, on trouve des blocs décimétriques déposés par les vagues jusqu’à $200 \mathrm{~m}$ à l'intérieur des terres. Une telle configuration est rare, même dans un environnement extrêmement énergétique comme celui du nord-est de l'Atlantique. Des dépôts de mégablocs liés à des vagues de tempêtes ont été identifiés à des distances équivalentes de la falaise mais à des altitudes supérieures à Villians of Hamnavoe aux îles Shetland (Hall et al., 2006). Toutefois, dans ce cas les dépôts les plus éloignés du trait de côte consistent en un épandage de graviers et cailloux. Sur les îles de la côte ouest de l'Irlande et de l'Écosse, les barrages de blocs sont en général plus proches du sommet des falaises, de quelques mètres à plus de $40 \mathrm{~m}$, mais ces dernières culminent

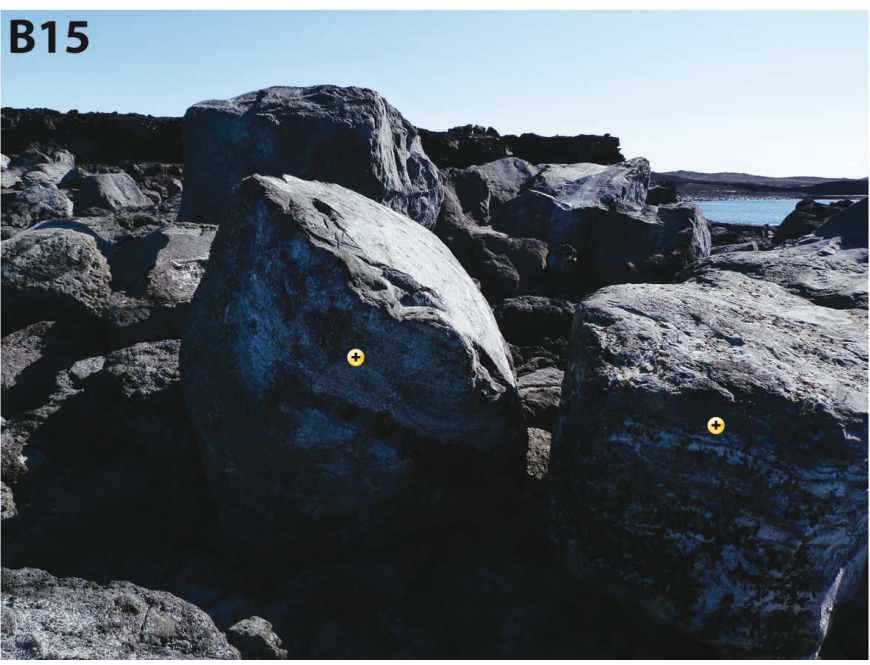

Fig 12 - Simplified geomorphological map of the cliff-top megaclast ridge of Kerling with associated profiles (A), five biggest megaclasts $a, b, c$ - axis and oblique photographs of 2014 (B14) and 2015 (B15).

1: cliff-top boulder garland; 2: megaclasts; 3: wave-scoured platform; 4: bedrock; 5: tephra; 6: cliff; 7: boulder beach; 8: wave-cut platform

toujours à plus de 15 m d'altitude (Hall et al., 2006, 2008). De telles configurations peuvent s'expliquer dans les cas où les vagues sont plus hautes que les falaises : soit à loccasion de très fortes tempêtes, soit lorsque les vagues incidentes sont amplifiées lors de leur rencontre avec une vague précédente réfléchie par la falaise (vagues scélérates ; Kharif et al., 2009), ou encore lorsque les vagues se superposent à des ondes infragravitaires (Sheremet et al., 2014). Hall et al. (2008) émettent l'hypothèse que dans certains cas la partie supérieure de ces vagues peut décoller en sommet de falaise (airborne waves) et atterrir plusieurs mètres en arrière où des blocs peuvent être arrachés à la roche en place, ou à leur support s'ils sont libres. Ces blocs sont ensuite projetés ou roulés vers l'arrière. Comme l'ont montré les travaux effectués sur lîle de Banneg (Finistère), ce processus permet 


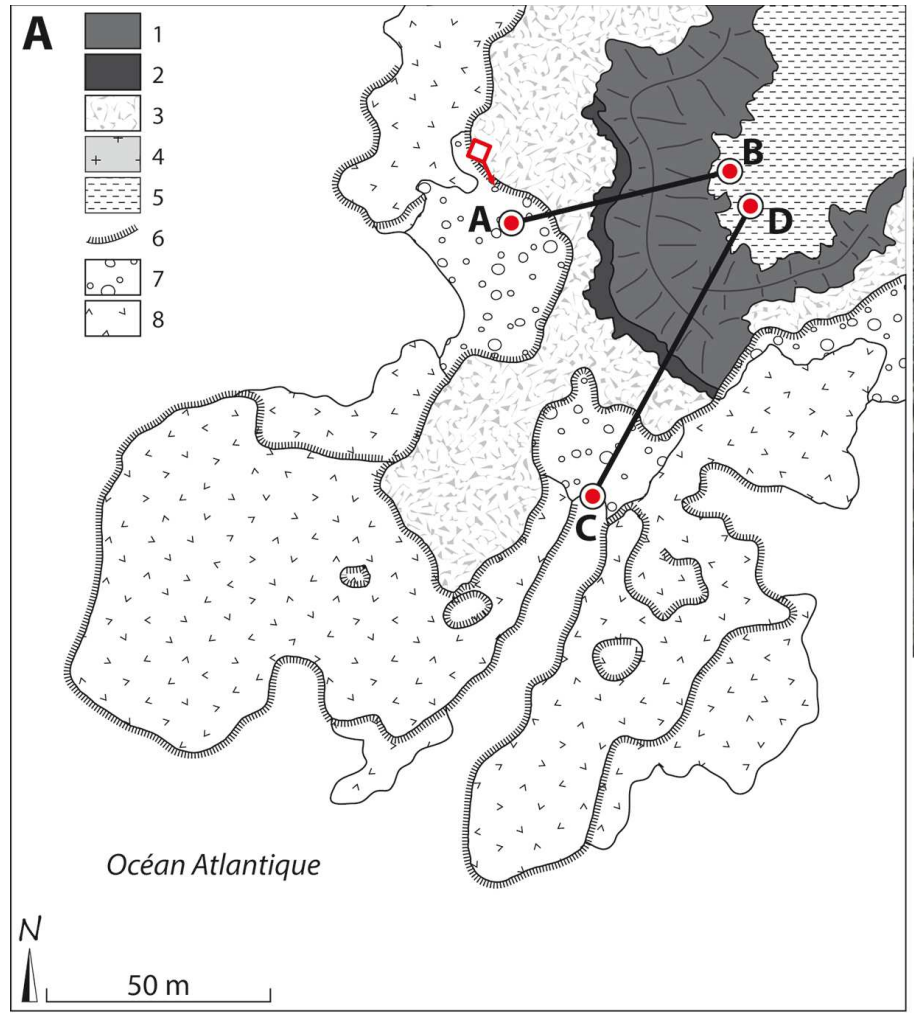

B

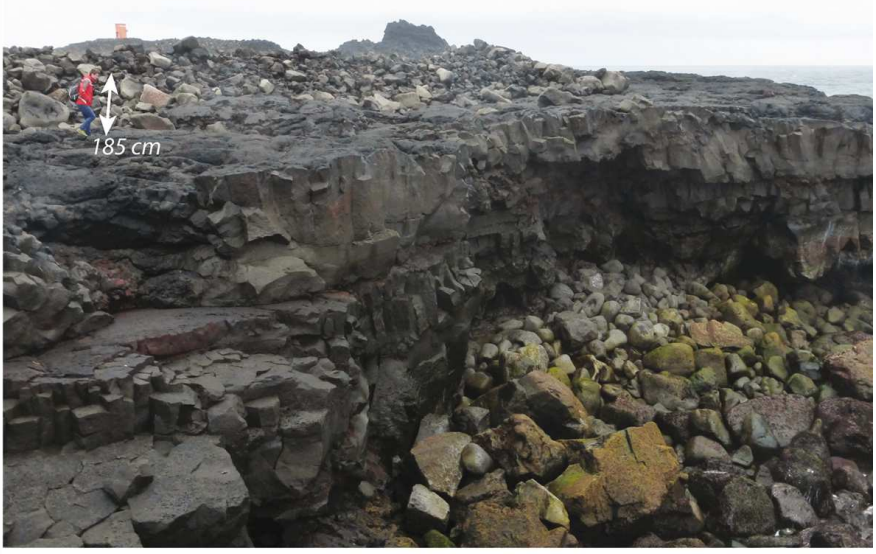

Profil $A-B$ :

axe $A$ axe $B$ axe $C$ Masse $(t)$ (b11) $285 \quad 192 \quad 147 \quad 22,5$ $\begin{array}{lllll}\text { (b12) } & 221 & 164 & 142 & 14,4\end{array}$ $\begin{array}{lllll}\text { (b13) } & 190 & 169 & 120 & 10,8\end{array}$ (b14) $\begin{array}{llll}173 & 142 & 80 & 5,5\end{array}$ (b15) $162 \quad 110 \quad 77 \quad 3,8$

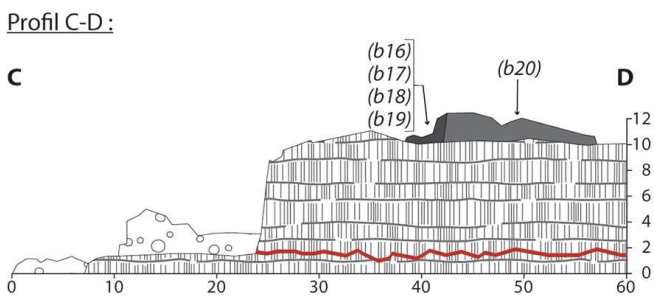

Fig 13 - Simplified geomorphological map of the cliff-top megaclast ridge of Reykjanestá with associated profiles (A), five biggest megaclasts $a, b, c$-axis and oblique photograph showing the site $(B)$.

1: cliff-top boulder garland; 2: megaclasts; 3: wave-scoured platform; 4: bedrock; 5: tephra; 6: cliff; 7: boulder beach; 8: wave-cut platform

sites sont comparables. La hauteur significative des vagues peut atteindre 18,5 $\mathrm{m}$ aux Shetlands et 17,4 $\mathrm{m}$ au large de la péninsule de Reykjanes, et l'isobathe $100 \mathrm{~m}$ se trouve dans les deux cas à environ deux kilomètres du trait de côte. Seule la configuration de la base des falaises diffère, falaises plongeantes dans le premier cas, précédées d'une plate-forme d'abrasion étroite dans le second. Toutefois, si des falaises hautes de 15 à $40 \mathrm{~m}$ peuvent être submergées par les vagues dans les îles britanniques, on imagine que le même phénomène peut aussi intervenir, et d'autant plus fréquemment, à Kerling, où les falaises ne culminent quà $8 \mathrm{~m}$. Cela expliquerait le dépôt de blocs beaucoup plus grossiers bien plus en arrière du bord de la falaise. Cependant, la submersion épisodique des falaises ne peut en aucun cas expliquer la présence de très gros blocs arrondis, voire de boules qui ont été façonnés sur les plates-formes d'abrasion avant de franchir l'abrupt des falaises. Notable à Kerling, ce type de dépôts est remarquable à Reykjanestá où la falaise verticale est haute de 8 à $10 \mathrm{~m}$. Il est sans doute extrêmement rare mais existe pourtant dans un contexte comparable à Hawaii en sommet de falaises basaltiques hautes de $5 \mathrm{~m}$, où de nombreuses boules, certes de petite taille, sont présentes (voir fig. 10B dans Richmond et al., 2011). Labondante littérature sur les modes d'arrachage et de transport de blocs, enchâssés ou pas dans la roche en place, névoque jamais ce cas de figure (Nott, 2003 ; partiellement la position du grand barrage de blocs de Kerling. En effet, les contextes hydrodynamique et bathymétrique de ces 
Noormets et al., 2004 ; Nandasena et al., 2013 ; Lau et al., 2015). Tous les modèles développés jusquà présent portent sur les forces requises pour essayer d'expliquer le transport par glissement, roulement ou saltation de masses rocheuses parfois considérables sur des surfaces plus ou moins inclinées, mais jamais verticales. Il est clair en tous cas que le déplacement vertical et de grande amplitude de blocs arrondis de plusieurs tonnes ne peut s'expliquer par la submersion du sommet de la falaise par des vagues géantes. En revanche, le déferlement de ces vagues en pied de falaise est une explication plus probable. Il reste alors à résoudre la question des conditions nécessaires à la remontée des boules. Bien que le saut des blocs à l'air libre sur de telles distances verticales ne soit pas à exclure, nous privilégions l'hypothèse d'une remontée « dans la masse » d'eau. Les lames d'eaux véloces et turbulentes venant sécraser contre la falaise à marée basse ou lorsque l'estran découvre en partie entre deux vagues provoquent un jaillissement d'eau vertical, emportant ainsi les boules mises en suspension.

\section{Remerciements}

Ce travail a bénéficié d'une aide de l'Etat gérée par l'Agence Nationale de la Recherche dans le cadre du LabexMer au titre du programme «Investissements d'avenir " portant la référence ANR10-LABX-19-01, et de la région Bretagne.

Nous remercions également Ingunn Erna Jónsdóttir, Jónas Hlíðar Vilhelmsson, et Sigurður Sigurðarson, du service «Icelandic Road and Coastal Administration (IRCA) " de Reykjavík, pour leur aide sur le terrain.

\section{Références}

Andrews J.T. (2005) - 2. Late Quaternary marine sediment studies of the iceland shelf - palaeocenography, land/ice sheet/ocean interactions, and deglaciation: a review. In: A.R.J. Harđardóttir and Ó. Knudsen C. Caseldine (dir.), Elsevier (Ed.). Developments in Quaternary Sciences, 5, 5-24. doi:10.1016/S1571-0866(05)80004-1

Bondevik S., Svendsen J.I., Mangerud J.A.N. (1997) - Tsunami sedimentary facies deposited by the Storegga tsunami in shallow marine basins and coastal lakes, western Norway. Sedimentology, 44, 1115-1131. doi:10.1046/j.1365-3091.1997.d01-63.x

Bondevik S., Mangerud J., Dawson S., Dawson A., Lohne $\emptyset$. (2005) - Evidence for three North Sea tsunamis at the Shetland Islands between 8000 and 1500 years ago. Quaternary Science Reviews, 24, 1757-1775. doi:10.1016/j.quascirev.2004.10.018

Charpentier J.G.F. de (1835) - Notice sur la cause probable du transport des blocs erratiques de la Suisse. Carilian-Goeury (Ed.), Paris, France.

Cox R., Zentner D.B., Kirchner B.J., Cook M.S. (2012) - Boulder Ridges on the Aran Islands (Ireland): Recent Movements Caused by Storm Waves, Not Tsunamis. The Journal of Geology, 120, 249-272. doi:10.1086/664787

Dawson A.G., Long D., Smith, D.E. (1988) - The Storegga slides: evidence from eastern Scotland for a possible tsunami. Marine geology, 82, 271-276. doi:10.1016/0025-3227(88)90146-6

Dawson A.G. (1999) - Linking tsunami deposits, submarine slides and offshore earthquakes. Quaternary International, 60 (1), 119-126. doi:10.1016/S1040-6182(99)00011-7

Dawson A.G., Shi S. (2000) - Tsunami Deposits. Pure and Applied Geophysics, 157, 875-897. doi:10.1007/s000240050010

Dionne J.-C. (2001) - Observations géomorphologiques sur les méga-blocs d'un schorre à Spartina alterniflora, estuaire maritime du Saint-Laurent, Québec / Geomorphic observations on the mega-boulders in a Spartina alterniflora tidal marsh, Lower St.
Lawrence estuary, Québec. Géomorphologie : relief, processus, environnement, 7, 243-255. doi:10.3406/morfo.2001.1109

Etienne S. (2007) - Les plates-formes d'érosion marine des littoraux volcaniques. In: Etienne S. et Paris R. (Ed.), Les littoraux volcaniques: une approche environnementale. Presses Univ Blaise Pascal, 37-55.

Étienne S., André M.-F. (2003) - La variabilité de la hiérarchie des processus de météorisation à travers les bilans météoriques de divers milieux périglaciaires nord-atlantiques (Islande, Labrador, Laponie, Spitsberg) / Variability in the hierarchy of weathering processes detected through weathering budgets of several NorthAtlantic periglacial environments (Iceland, Labrador, Lapland, Spitsbergen). Géomorphologie : relief, processus, environnement, 9, 177-189. doi:10.3406/morfo.2003.1178

Etienne S., Paris R. (2010) - Boulder accumulations related to storms on the south coast of the Reykjanes Peninsula (Iceland). Geomorphology, 114, 55-70. doi:10.1016/j.geomorph.2009.02.008

Fichaut B., Suanez S. (2008) - Les blocs cyclopéens de l'île de Banneg (archipel de Molène, Finistère): accumulations supratidales de forte énergie. Géomorphologie : Relief. Processus. Environnement, 1, 15-32. doi:10.4000/geomorphologie.5793

Fichaut B., Suanez S. (2011) - Quarrying, transport and deposition of cliff-top storm deposits during extreme events: Banneg Island, Brittany. Marine Geology, 283, 36-55. doi:10.1016/j. margeo.2010.11.003

Goto K., Miyagi K., Kawana T., Takahashi J., Imamura F. (2011) Emplacement and movement of boulders by known storm waves Field evidence from the Okinawa Islands, Japan. Marine Geology, 283, 66-78. doi:10.1016/j.margeo.2010.09.007

Gudmundsson A. (1987) - Geometry, formation and development of tectonic fractures on the Reykjanes Peninsula, southwest Iceland. Tectonophysics, 139, 295-308. doi:10.1016/0040-1951(87)90103-X

Hall A.M., Hansom J.D., Williams D.M., Jarvis J. (2006) Distribution, geomorphology and lithofacies of cliff-top storm deposits: Examples from the high-energy coasts of Scotland and Ireland. Marine Geology, 232, 131-155. doi:10.1016/j. margeo.2006.06.008

Hall A.M., Hansom J.D., Jarvis J. (2008) - Patterns and rates of erosion produced by high energy wave processes on hard rock headlands: The Grind of the Navir, Shetland, Scotland. Marine Geology, 248, 28-46. doi:10.1016/j.margeo.2007.10.007

Hansom J.D., Hall A.M. (2007) - Magnitude and frequency of extratropical North Atlantic cyclones: A chronology from cliff-top storm deposits. Quaternary International, 195, 42-52. doi:10.1016/j. quaint.2007.11.010

Hansom J.D., Barltrop N.D.P., Hall A.M. (2008) - Modelling the processes of cliff-top erosion and deposition under extreme storm waves. Marine Geology, 253, 36-50. doi:10.1016/j. margeo.2008.02.015

Hartley A., Howell J., Mather A.E., Chong G. (2001) - A possible Plio-Pleistocene tsunami deposit, Hornitos, northern Chile. Revista geológica de Chile, 28, 117-125. doi:10.4067/S071602082001000100007

Hearty P.J. (1997) - Boulder Deposits from Large Waves during the Last Interglaciation on North Eleuthera Island, Bahamas. Quaternary Research, 48, 326-338. doi:10.1006/qres.1997.1926

Jones B., Hunter I.G. (1992) - Very Large Boulders on the Coast of Grand Cayman: The Effects of Giant Waves on Rocky Coastlines. Journal of Coastal Research, 8, 763-774.

Kelletat D., Schellmann G. (2002) - Tsunamis on Cyprus: field evidences and $14 \mathrm{C}$ dating results. Zeitschrift Für Geomorphologie, 46, 19-34. 
Kharif C., Pelinovsky E., Slunyaev A. (2009) - Rogue waves in the ocean. Springer (Ed.), Berlin, 173-209.

Knight J., Burningham H. (2011) - Boulder dynamics on an Atlantic-facing rock coastline, northwest Ireland. Marine Geology, 283, 56-65. doi:10.1016/j.margeo.2010.07.008

Knight J., Burningham H., Barrett-Mold C. (2009) - The geomorphology and controls on development of a boulderstrewn rock platform, NW Ireland. Journal of Coastal Research, SI 56 (Proceedings of the $10^{\text {th }}$ International Coastal Symposium), 1646-1650.

Kogure T., Matsukura Y. (2010) - Instability of coral limestone cliffs due to extreme waves. Earth Surface Processes and Landforms, 35, 1357-1367. doi: 10.1002/esp.2046

Lau A.A., Terry J.P., Switzer A.D., Pile J. (2015) - Advantages of beachrock slabs for interpreting high-energy wave transport: Evidence from Ludao Island in south-eastern Taiwan. Geomorphology, 228, 263-274. doi:10.1016/j. geomorph.2014.09.010

Long D., Smith, D.E., Dawson A.G. (1989) - A Holocene tsunami deposit in eastern Scotland. Journal of Quaternary Science, 4, 6166. doi:10.1002/jqs.3390040107

Marie G. (2004) - L'évolution des formes et de la morphodynamique en domaine littoral volcanique : recherches dans l'archipel d'Hawaï. 522p. Thèse de doctorat. Université de Bretagne occidentale - Brest.

Miller S., Rowe D.-A., Brown L., Mandal A. (2013) - Waveemplaced boulders: implications for development of " prime real estate " seafront, North Coast Jamaica. Bulletin of Engineering Geology and the Environment, 73, 109-122. doi:10.1007/s10064013-0517-0

Moign A., Moign Y. (1970) - Les îles Heimaey et Surtsey de l’archipel volcanique Vestmannaejar (Islande). Étude du littoral. Norois, 67, 305-334. doi:10.3406/noroi.1970.7339

Moore J.G., Moore, G.W. (1984) - Deposit from a Giant Wave on the Island of Lanai, Hawaii. Science, 226, 1312-1315. doi:10.1126/ science.226.4680.1312

Nakata, T., Kawana, T. (1995) - Historical and prehistorical large tsunamis in the southern Ryukyus, Japan. In Tsunami: Progress in Prediction, Disaster Prevention and Warning (pp. 211-221). Springer Netherlands.

Nandasena N.A.K., Tanaka N., Sasaki, Y., Osada M. (2013) Boulder transport by the 2011 Great East Japan tsunami: Comprehensive field observations and whither model predictions? Marine Geology, 346, 292-309. doi:10.1016/j.margeo.2013.09.015

Noormets R., Crook K.A.W., Felton E.A. (2004) - Sedimentology of rocky shorelines: 3. Hydrodynamics of clast emplacement and transport on a shore platform, Oahu, Hawaii. Sedimentary Geology, 172, 41-65. doi:10.1016/j.sedgeo.2004.07.006

Nott J. (1997) - Extremely high-energy wave deposits inside the Great Barrier Reef, Australia: determining the cause-tsunami or tropical cyclone. Marine Geology, 141, 193-207. doi:10.1016/ S0025-3227(97)00063-7

Nott J. (2003) - Waves, coastal boulder deposits and the importance of the pre-transport setting. Earth and Planetary Science Letters, 210, 269-276. doi:10.1016/S0012-821X(03)00104-3

Paskoff R. (1991) - Likely occurrence of a mega-tsunami in the Middle Pleistocene, near Coquimbo, Chile. Andean Geology, 18, 87-91. doi:10.5027/andgeoV18n1-a08

Peterson D.W., Tilling R.I. (1980) - Transition of basaltic lava from pahoehoe to aa, Kilauea Volcano, Hawaii: Field observations and key factors. Journal of Volcanology and Geothermal Research, 7, 271-293. doi:10.1016/0377-0273(80)90033-5

Prizomwala S.P., Gandhi D., Ukey V.M., Bhatt N., Rastogi, B.K. (2014) - Coastal boulders as evidences of high-energy marine events from Diu Island, west coast of India: storm or palaeotsunami? Natural Hazards, 75, 1187-1203.

Richmond B.M., Watt S., Buckley M., Jaffe B.E., Gelfenbaum G., Morton, R.A. (2011) - Recent storm and tsunami coarse-clast deposit characteristics, southeast Hawaii. Marine Geology, 283, 79-89. doi:10.1016/j.margeo.2010.08.001

Russell A.J., Fay H., Marren P.M., Tweed F.S., Knudsen Ó. (2005) 7. Icelandic jökulhlaup impacts. In: A.R.J. Harđardóttir and Ó. Knudsen C. Caseldine (dir.), Elsevier (Ed.), Developments in Quaternary Sciences, 5, 153-203.

Salzmann L., Green A. (2012) -. Boulder emplacement on a tectonically stable, wave-dominated coastline, Mission Rocks, northern KwaZulu-Natal, South Africa. Marine Geology, 323-325, 95-106. doi:10.1016/j.margeo.2012.07.001

Scheffers A., Scheffers S., Kelletat D., Browne T. (2009) - WaveEmplaced Coarse Debris and Megaclasts in Ireland and Scotland: Boulder Transport in a High-Energy Littoral Environment. The Journal of Geology, 117, 553-573. doi:10.1086/600865

Shah-Hosseini M., Morhange C., Naderi Beni A., Marriner N., Lahijani H., Hamzeh M., Sabatier F. (2011) - Coastal boulders as evidence for high-energy waves on the Iranian coast of Makran. Marine Geology, 290, 17-28. doi:10.1016/j.margeo.2011.10.003

Shah-Hosseini M., Morhange C., De Marco A., Wante J., Anthony E.J., Sabatier F., Mastronuzzi G., Pignatelli C., Piscitelli A. (2013) - Coastal boulders in Martigues, French Mediterranean: evidence for extreme storm waves during the Little Ice Age. Zeitschrift für Geomorphologie, Supplement, 4, 181-199. doi:10.1127/0372-8854/2013/S-00132

Sheremet A., Staples T., Ardhuin F., Suanez S., Fichaut B. (2014) - Observations of large infragravity wave runup at Banneg Island, France. Geophysical Research Letters, 41, 976-982. doi:10.1002/2013GL058880

Sigurgeirsson M. (1995) - Yngra-Stampagosid a Reykjanesi (The Younger-Stampa eruption at Reykjanes, SW-Iceland). Náttúrufraedhingurinn, 64, 211-230.

Suanez S., Fichaut B., Magne R. (2009) - Cliff-top storm deposits on Banneg Island, Brittany, France: Effects of giant waves in the Eastern Atlantic Ocean. Sedimentary Geology, 220, 12-28. doi:10.1016/j.sedgeo.2009.06.004

Sunamura, T. (1988) - Beach morphologies and their change. In: Horikawa, K. (Ed.). Nearshore Dynamics and Coastal Processes. University of Tokyo Press, Tokyo, pp. 135-161.

Switzer A.D., Burston, J.M. (2010) - Competing mechanisms for boulder deposition on the southeast Australian coast. Geomorphology, 114, 42-54. doi:10.1016/j.geomorph.2009.02.009

Terry J.P., Goff J. (2014) - Megaclasts: Proposed Revised Nomenclature At the Coarse End of the Udden-Wentworth GrainSize Scale for Sedimentary Particles. Journal of Sedimentary Research, 84, 192-197. doi:10.2110/jsr.2014.19

Thordarson T., Larsen G. (2007) - Volcanism in Iceland in historical time: Volcano types, eruption styles and eruptive history. Journal of Geodynamics, 43, 118-152. doi:10.1016/j.jog.2006.09.005

Whelan F., Keating B. (2004) - Tsunami deposits on the island of Oahu, Hawaiii. In Schernewski G., Dolch T. (Eds.): Geographie der Meere und Küsten, Coastline Reports, 1, 77-82.

Williams D.M., Hall A.M. (2004) - Cliff-top megaclast deposits of Ireland, a record of extreme waves in the North Atlanticstorms or tsunamis? Marine Geology, 206, 101-117. doi:10.1016/j. margeo.2004.02.002

Young R.W., Bryant E.A. (1992) - Catastrophic wave erosion on the southeastern coast of Australia: Impact of the Lanai tsunamis ca. 105 ka? Geology, 20, 199-202. doi:10.1130/00917613(1992)020<0199:CWEOTS >2.3.CO;2 


\section{English abridged version}

The present study analyses cliff-top storm deposits (CTSD) in the Reykjanes peninsula which corresponds to the emerged part of the midAtlantic ridge in the southwest of Iceland (fig. 1). The $35 \mathrm{~km}$ of coast studied mainly consists of cliffs 4 to $18 \mathrm{~m}$ high $\mathrm{s}$ cut into postglacial basaltic lava flows (750 to 14,500 years old). Consequently clasts on top of cliffs cannot be erratics, or derived from tsunamis as none have been reported in this area in this time lapse. This area facing deep water is a high-energy marine environment, with more than 50 storm days per year. The maximum significant wave height reach $17.4 \mathrm{~m}$ (fig. 3) and water depths reach $100 \mathrm{~m}$ within 2 to 3 kilometres of the shore. Morphological and sedimentary measurements were carried on CTSD (individual, clusters or ridges) occurring in 35 sites (fig. 4).

The morphosedimentary dynamics have been characterized through analysis of oblique photos taken from 2007 to 2015 (fig. 5). The typology derived for these deposits (fig. 6) is based on various parameters: (i) the structure of the bedrock, which always consists of horizontal or sub-horizontal lava beds with variable thickness and surface structures (fig. 2), (ii) the morphology of shore platforms, cliffs and wave-scoured platforms, (iii) the local hydrodynamic context. Isolated clasts occur seldom on the highest cliffs (fig. 7).

CTSD resulting from the erosion of the surface layer of pahoehoe lava beds form discontinuous chains of ridges roughly parallel to the cliff top several tens of metres inland (fig. 8-9A). 60 to $80 \%$ of the clasts are small $(0.25<b$ axis $<0.5 \mathrm{~m})$, with the rest between $0.5<b$ axis $<$ $2 \mathrm{~m}$. The low density of joints in the inside parts of lava beds render them more resistant to wave action and the wave-scoured platform is generally smooth and swept clear of debris by storm wave activity (fig. 9A1-2). However prismatic boulders derived from the detachment of clasts on the cliff top or caves on the platform occur seldom in this type of accumulation.

CTSD resulting from the erosion of 'a'a lava beds are more voluminous, characterized by a heterogeneous granulometry (fig. 9B). Scattered boulders of all sizes abound in the crevices of the rough and jagged wave-scoured platform (fig. 9B1-2). Some CTSD contain as much as 40 to $60 \%$ of rounded boulders, which proves that they stayed long enough in the intertidal area before being transported over the cliff top. Locally they occur on top of vertical cliffs when the lava beds are several metres thick (fig. 10A, A1-3). Sometimes they are located in accumulations occurring in the back of staircase cliffs or ramps cut into several piledup lava beds (fig. 10B-1).

In this case, wave action is favoured by the slope, and rounded boulders may represent over $70 \%$ of the CTSD. CTSD containing megaclasts, including rounded ones weighing several tonnes, occur only on two sites that are the most energetic of the peninsula (fig. 11). The Kerling ridge (fig. 12A), $180 \mathrm{~m}$-long and $60 \mathrm{~m}$-wide, stands $80 \mathrm{~m}$ from the edge of 6 to $8 \mathrm{~m}$-high cliffs. Its crest is $10 \mathrm{~m}$ a.s.l., and decimetric clasts may be found $200 \mathrm{~m}$ inland on the back of the ridge. Huge boulders were deposited up to $60 \mathrm{~m}$ inland on the rough wave-scoured platform during the 2014-2015 winter (fig. 12B). Here among other parameters, extreme wave energy accounts for the position and volume of the ridge as well as the occurrence of these very large boulders. The ridge of Reykjanestá is located 8 to $10 \mathrm{~m}$ a.s.l. in the back of a 15 to $20 \mathrm{~m}$-wide platform (fig. 13A, profile $A-B$ ). The specificity of this CTSD lies in the fact that many of the very large boulders present are rounded and were transported from the base to the top of a vertical cliff 6 to $8 \mathrm{~m}$-high cliff. It is obvious that these clasts did not make their way up progressively but were transported all of a sudden by extreme waves breaking at the base of the cliff. The discussion focuses on the importance of the structure of the lava beds to explain the morphology of some CTSD.

It also highlights the fact that very large CTSD containing mega boulders may be formed in the back of very large cliff-top platforms by storm waves. It also brings up the fact that all existing models related to boulder transportation on or over cliff tops deal with sliding, rolling or saltation, but never vertical high jumps. 